\title{
A study of the vibration of a horizontal U-bend subjected to an internal upwards flowing air-water mixture
}

\author{
Laurent De Moerloose ${ }^{\mathrm{a}, *}$, Joris Degroote $\mathrm{e}^{\mathrm{a}, \mathrm{b}}$ \\ ${ }^{a}$ Department of Flow, Heat and Combustion Mechanics, Faculty of Engineering and \\ Architecture, Ghent University, Sint-Pietersnieuwstraat 41-B4, 9000 Ghent, Belgium \\ ${ }^{b}$ Flanders Make, Belgium
}

\begin{abstract}
U-bends are a common geometry in heat exchangers. In this paper, a U-bend in the vertical plane connected to horizontal straight pipes is considered. An initially stratified water/air flow moves upwards against gravity. The aim of this research is to investigate the internal flow profile and resulting force when the U-bend is subjected to a stratified air-water flow at the inlet. This is done numerically, i.e. by solving the unsteady Reynolds-averaged Navier-Stokes equations. For low mass flow rates, large gas bubbles are naturally formed at the entrance of the bend. The transient force on the tube allows to determine precisely the time instants of bubble initiation and thus to quantify the bubble frequency. Firstly, the tube is assumed to be rigid and the dependence of force oscillation on the inlet conditions is investigated. Secondly, the influence of the viscosity, wall wetting and the mass flow rate is analyzed. Finally, a fluidstructure interaction calculation is performed in order to quantify the vibration characteristics of the tube.
\end{abstract}

Keywords: two-phase flow, U-bend, computational fluid dynamics, fluid-structure interaction

\section{Introduction}

U-bends are commonly encountered in many heat exchanger geometries (Kakaç et al. (2012)), such as shell-and-tube heat exchangers. When these heat exchangers are used as evaporator, the tubes are subjected to two-phase flow (water and steam). A lot of research has been done on the vibration of Ubends subjected to external two-phase flow, mainly driven by the application in the nuclear plants (Yetisir and Weaver (1986); Weaver and Fitzpatrick (1988); Pettigrew and Taylor (2003a,b)). Consequently, the vibration characteristics

\footnotetext{
*Corresponding author. Tel. +32 926432 97, Fax. +3292643590

Email address: laurent.demoerloose@ugent.be (Laurent De Moerloose)
} 
of a tube bundle with or without U-bend subjected to external two-phase flow are relatively well understood, although not completely (Pettigrew and Janzen (2016)). Pettigrew et al. (1998) found that the vibration amplitude decreases with increasing average pressure and proposed a number of correlations for the damping coefficients and dominant frequencies. In contrast, despite the abundance of applications of U-bends subjected to internal two-phase flow, little research can be found about the vibration of this system. Most of the research on internal two-phase flow focuses on the vibrations of straight pipes (Lannes and Gama (2018)). Nakamura et al. (1995) performed experiments with air-water and steam-water flow and concluded that the vibrations in these geometries are closely related to the impact of liquid slugs on the pipes. The excitation force in the air-water and steam-water flow were found to be similar. Finally, the maximal vibration amplitude was proportional to the slug speed and thus to the mass flux. Geng et al. (2012) found both analytically and numerically that the frequency of the tube vibration and the energy content of the flow is severely dependent on the flow regime. They therefore suggested that the vibration characteristics of a straight pipe could be used to identify the flow regime inside the pipe. However, their experiments also revealed that the pipe vibration is heavily dependent on the mass flux as well: the standard deviation of the vibration increases with both liquid and gas flow rate and a vibration amplitude increase is recorded for increasing gas flow rate. Therefore, the proposed identification is not easily performed and has not been fully developed yet. Charreton et al. (2015) investigated the damping coefficient for internal two-phase flow through a vertical pipe and proposed a similar decomposition of the damping coefficient as Pettigrew and Taylor (2003a) did for external flow.

Specific bend structures are also found in literature, for example in the work of Belfroid et al. (2016). From this, it is clear that the period and the shape of the transient force profile on a bend subjected to internal two-phase flow can be described by summing the pressure and momentum terms, even though the amplitude of the highest force peaks is underpredicted by such a model. Similar to what was discussed by Nakamura et al. (1995), the impact by liquid slugs cannot be neglected. It should be noted that Mack et al. (2018) validated the experimental work described by Belfroid et al. (2016) and confirmed the conclusions.

The bends subjected to two-phase flow described in literature vary in size and geometry, but the current research on U-bends specifically is mainly subdivided into two parts. On the one hand, a lot of data can be found about the global pressure drop over a U-bend, typically relating this variable to the measured or imposed void fraction and mass flux found in the bend. De Kerpel et al. (2012) report the pressure drop in such manner, with R134a as working fluid. They conclude that the flow is not fully-developed after 30 diameters. Another observation is that the U-bend does not seem to affect to the flow profile in the inlet tube until just in front of it. The latter observation is interesting as the numerical domain does not have to be excessively long. On the other hand, some work was done to investigate the exact liquid-gas distribution inside a U-bend. Da Silva Lima and Thome (2012) performed experiments on both horizontally 
and vertically positioned U-bends over a broad range of tube diameters, bend radii and mass flow rates. For low mass flow rates, the liquid layer is pushed initially to the outer wall of the bend (due to gravity/buoyancy), whereas more annular flow is obtained for higher mass flow rates. In any case, the liquid and gas phases are clearly separated by a continuous interface, with little droplet entrainment in the gas phase and gas entrapment in the liquid phase. Usui et al. (2012) report similar findings and define a characteristic Froude number to quantify the transition from a gravity-dominant to an inertia-dominant flow. Wang et al. (2005) and Wang et al. (2008) investigated the behavior of a water-air mixture inside a small-diameter U-bend, but did not perform force calculations or vibrations measurements.

This paper calculates the water-air distribution inside a U-bend and relates the distribution to the transient force and displacement imposed on the Ubend. To the best of the authors' knowledge, there has been no numerical investigation on the transient force on and the vibration of a U-bend subjected to an internal air-water flow. Experimental data of the same geometry is very scarce in literature as well. The goal of this research is not to model a geometry used in an actual steam generator geometry, but to present a methodology that can be used on an actual geometry in future work on the one hand and to apply the described procedure on a particular U-bend geometry to gain insight into the fundamental flow behavior on the other hand. This insight can be useful for understanding two-phase flow at low velocities, e.g. in small heat exchangers. The return bend considered in the paper is purely based on the work by Wang et al. (2008) and is positioned in the vertical plane, but the inlet and outlet tube are horizontal. Firstly, this geometry is used in a Unsteady ReynoldsAveraged Navier-Stokes calculation in order to obtain the force profile inside a rigid tube. The effect of the inlet profile, the liquid viscosity, the wall wetting angle, the mass flow rate and the operating density is investigated. Additionally, simulations of compressible flow inside a rigid U-bend are presented. In the second part of this paper, a linear elastic tube is modelled with a finite-element structural solver and coupled to the flow solver in order to perform a partitioned fluid-structure interaction simulation of the U-bend and to visualize and analyze the displacement of the U-bend. 


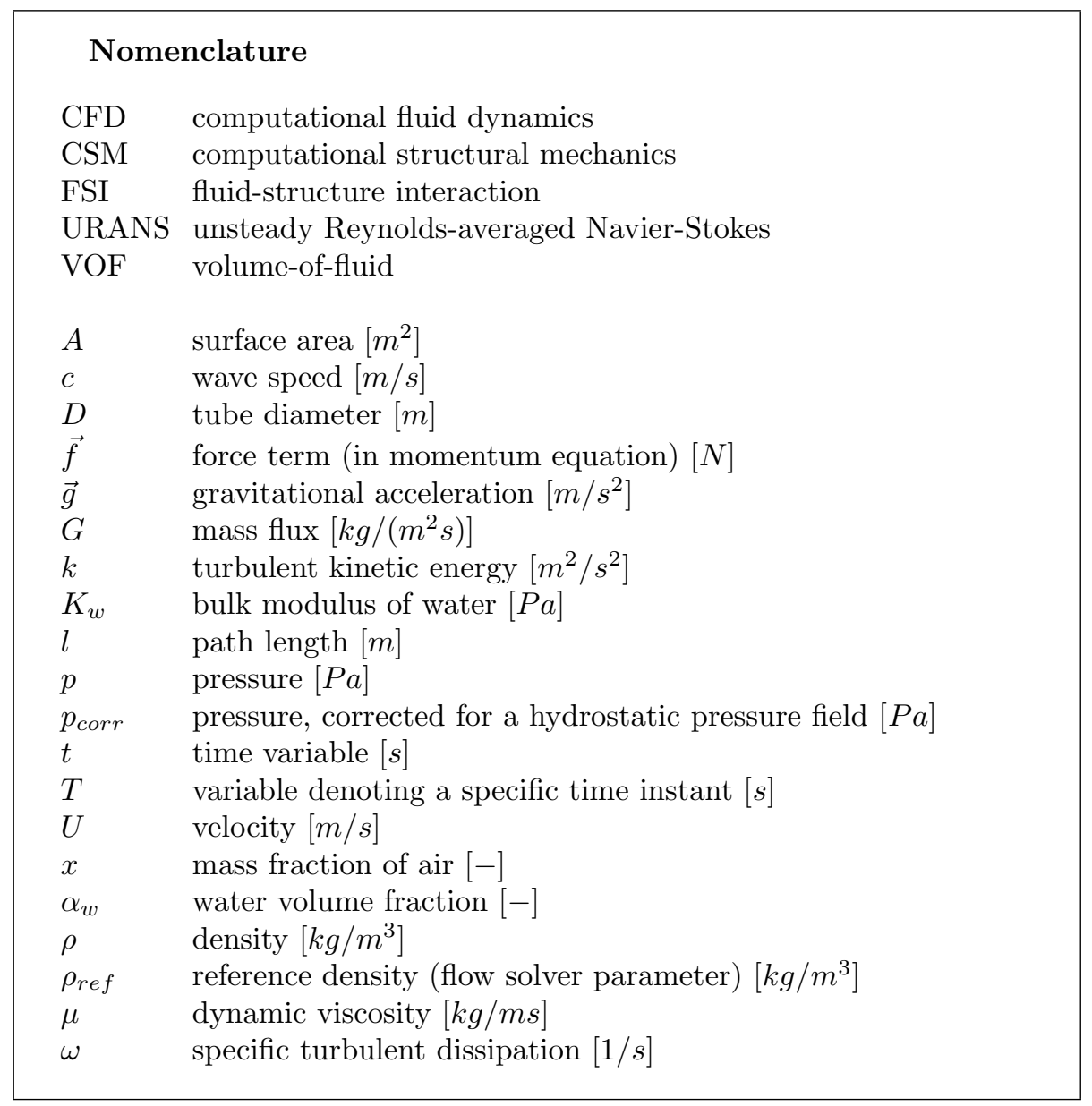

\section{Method}

\subsection{U-bend geometry}

The U-bend geometry is based on the work by Wang et al. (2005) and is shown in Figure 1. The flow enters and leaves the domain in the horizontal direction. Since the inlet is located below the outlet, the fluid inside the U-bend is moving upwards against gravity. The pipe diameter is denoted by $D$ and equals $0.0069 \mathrm{~m}$. The inlet and outlet tube are respectively $5 D$ and $4 D$ long, with the bend radius being equal to $1.5 \mathrm{D}$. The dimensions of the inlet and outlet tube are deemed acceptable based on the previously discussed observation of De Kerpel et al. (2012) that the U-bend does not significantly affect the flow profile, except for a few diameters in front of it. However, it should be noted that the flow does not have sufficient place to fully develop. In some simulations, fullydeveloped flow is required. Whenever this is the case, an inlet tube of length 
$200 D$ is appended to the domain. The cross-sectional view of the mesh is kept the same as in the reference U-bend mesh (see Figure $2 \mathrm{~b}$ ). The total cell count of the mesh in the straight horizontal tube is 125,000 . This prolonged inlet pipe is applied to the simulations discussed in Section 4.4 and Section 4.5.

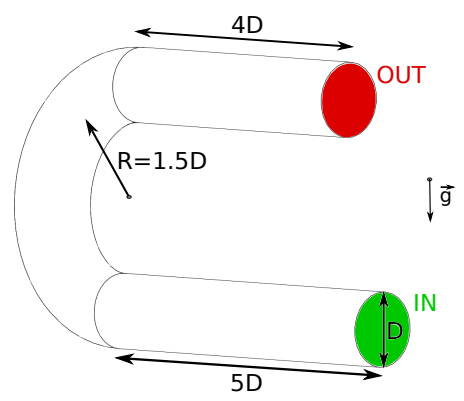

Figure 1: Schematic view of the U-bend.

The mesh used in most numerical simulations contains 630,000 cells. A midplane and cross-sectional view of this mesh are shown in Figure 2. A mesh sensitivity study is presented in Section 3 .

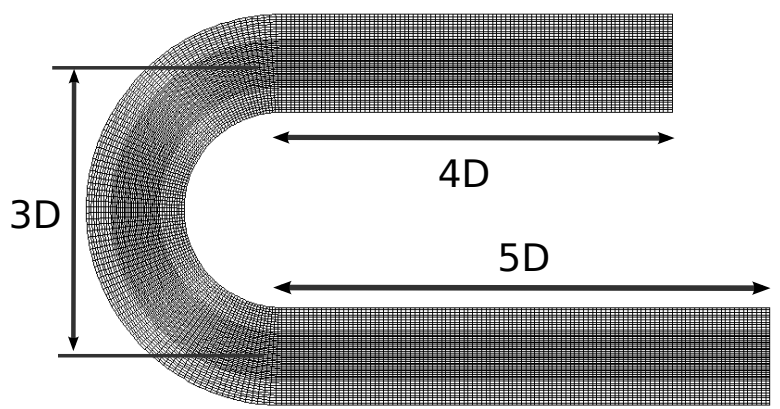

(a)

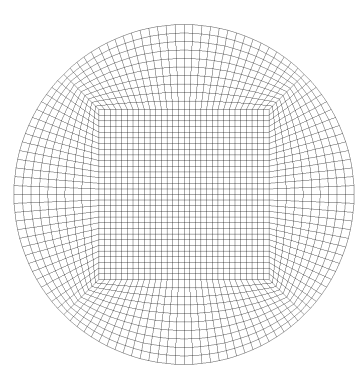

(b)

Figure 2: View of the reference mesh containing 630,000 cells. (a) Midplane (b) Cross-section of the tube.

\subsection{Fluid and structure parameters}

In the present numerical simulations, the working fluid is a water-air mixture. The densities of water $\left(\rho_{w}\right)$ and air $\left(\rho_{a}\right)$ are equal to $1000 \mathrm{~kg} / \mathrm{m}^{3}$ and $1.205 \mathrm{~kg} / \mathrm{m}^{3}$, respectively. The liquid viscosity $\left(\mu_{w}\right)$ is set to $1 \mathrm{e}-03 \mathrm{~kg} / \mathrm{ms}$, whereas the gas viscosity $\left(\mu_{a}\right)$ has a value of $18.21 \mathrm{e}-06 \mathrm{~kg} / \mathrm{ms}$. The surface tension between both phases $(\sigma)$ is equal to $0.07275 \mathrm{~N} / \mathrm{m}$. Except in Section 4.3 where the effect 
of liquid viscosity is investigated, these parameters are used in all simulations in the present study.

In the structural model, PVC is used as material. The material behaves linear elastically, the density, Young's modulus, Poisson's ratio are defined as $1400 \mathrm{~kg} / \mathrm{m}^{3}, 3.15 \mathrm{GPa}$ and 0.4 , respectively.

\subsection{Models}

\subsubsection{Computational Fluid Dynamics}

In the computational fluid dynamics (CFD) simulations performed on the two-phase flow in a rigid tube, the fluid is modelled with a one-fluid approach. This means that only one mass and one momentum equation is solved on the mixture level. Therefore, the interaction between the different phases is not modelled, except the non-zero forces on the liquid-gas interface (e.g. surface tension). The classical Volume-Of-Fluid (VOF) technique is used. In this approach, the mass and momentum equation are given by:

$$
\begin{gathered}
\frac{\partial \rho_{m}}{\partial t}+\nabla \cdot\left(\rho_{m} \vec{U}\right)=0 \\
\frac{\partial \rho_{m} \vec{U}}{\partial t}+\nabla \cdot\left(\rho_{m} \vec{U} \vec{U}\right)=\rho_{m} \vec{g}-\nabla p+\vec{f}+\nabla \cdot \mu\left(\nabla \vec{U}+\nabla \vec{U}^{T}\right)-2 \sigma \frac{\rho_{m}}{\rho_{w}+\rho_{a}} \nabla \cdot\left(\frac{\nabla \alpha_{w}}{\left|\nabla \alpha_{w}\right|}\right) \nabla \alpha_{w}
\end{gathered}
$$

where $\alpha_{w}$ represents the cell volume fraction of water. It should be noted that the Continuum Surface Force method (Brackbill et al. (1992)) was used: the force related to the surface tension is equal to $\sigma \kappa \vec{n}$, with $\kappa$ the curvature and $\vec{n}$ the normal vector on the liquid-gas interface. As such, the vector $\vec{n}$ is replaced by $\nabla \alpha_{w}$ and the curvature $\kappa$ is defined as $-\nabla \cdot\left(\nabla \alpha_{w} /\left|\nabla \alpha_{w}\right|\right)$. The fluid density and viscosity are mixture variables (as indicated by the index $m$ ), meaning they are a weighted average of the water and air properties, with $\alpha_{w}$ as weighting function:

$$
\begin{aligned}
\rho_{m} & =\alpha_{w} \rho_{w}+\left(1-\alpha_{w}\right) \rho_{a} \\
\mu_{m} & =\alpha_{w} \mu_{w}+\left(1-\alpha_{w}\right) \mu_{a}
\end{aligned}
$$

in which the indices $w$ and $a$ denote the properties of water and air, respectively.

An additional equation is required to completely define the problem. In an incompressible flow problem, the transport equation for the volume fraction $\alpha_{w}$ is reduced to the simple form:

$$
\frac{\partial \alpha_{w}}{\partial t}+\nabla \cdot\left(\alpha_{w} \vec{U}\right)=0
$$

As turbulence is modelled with the $k-\omega$ Shear Stress Transport (SST) model, two additional differential equations for $k$ and $\omega$, respectively, have to be solved. These equations are the same in a one-fluid model as these defined for the single-phase case (Menter (1994)).

In order to guarantee a stable solution, a pressure-based solver with fullycoupled approach was applied. As such, the mass, momentum, turbulence equations and the scalar transport equation for $\alpha_{w}$ were solved in a coupled manner. 
The convective and pressure terms were spatially discretized with the secondorder upwind and PRESTO!-schemes, respectively. The PRESTO!-scheme calculates the face value of the pressure directly from a discretized continuity balance applied on a staggered control volume. The gradients are calculated with a least-squares cell-based approach, i.e. the system of equations relating the cell gradient to the difference in cell values is minimized with a least-squares model. The compressive scheme was used for the interpolation of $\alpha_{w}$. This interface reconstruction scheme calculates the face values of $\alpha_{w}$ from summing the cell centre value with the cell's $\alpha_{w}$ gradient value multiplied with a slope limiter value equal to 2 . The implementation in Fluent is based on the work by Ubbink (1997), but is adapted to accommodate implicit time discretization. In order to limit the computational time, the first order upwind scheme was used to discretize the turbulence quantities $k$ and $\omega$. This is acceptable from an accuracy point of view, because turbulence will hardly affect the flow profile in the present simulations, as discussed in Section 3.2. The residuals of all equations except the continuity equation dropped below 1e-05 during each time step. For each flow equation independently, the residual is expressed as the ratio of the sum of the absolute errors in each cell to the sum of the absolute values of the flow variable calculated in each cell. Both the nominator and the denominator are re-evaluated in each iteration. The residual of the continuity equation is expressed in a different way, i.e. as the ratio of the sum of the rate of mass creation in each cell to the largest absolute value of this sum in the first five iterations of a time step. The final continuity residual fluctuates between 1e-04 and $1 \mathrm{e}-03$ in each time step. Finally, the time derivatives are discretized with a second-order implicit scheme with variable time step. The time step is set to $1 \mathrm{e}-04 \mathrm{~s}$, except in the vicinity of the occurrence of force peaks, when it is lowered to $5 \mathrm{e}-06 \mathrm{~s}$.

A stratified inlet profile is applied at the inlet, where the water is located at the bottom of the pipe and the air is positioned on top of it. A no-slip condition is applied to the tube wall. The outlet is defined as a pressure outlet, where a corrected pressure $p_{\text {corr }}$ is set to zero. This pressure is defined as:

$$
p_{\text {corr }}=p-\rho_{\text {ref }} g h
$$

where $g$ is the gravitational constant $\left(9.81 \mathrm{~m} / \mathrm{s}^{2}\right)$ and $h$ is the difference in height to a reference point. For the CFD calculations, the reference density is set to the initial volume-average of the density in the domain, i.e. the weighted sum of the mixture density defined in Equation (3a) with the cell volume as weighting function. This means that the hydrostatic pressure is not exactly modelled at the outlet, since the amount of water at the outlet changes over time. This causes a slight acceleration of the flow at the outlet, as will be discussed in Section 4.2 , but this is a local phenomenon.

The software code ANSYS ${ }^{\circledR}$ Fluent ${ }^{\circledR} 17.1$ was used for the CFD simulations presented in this paper, except in Section 4.7 where version 18.2 was used for the compressible flow calculations. 


\subsubsection{Fluid-Structure Interaction}

Following the aforementioned CFD analysis, the vibrations in a U-bend are analyzed by numerically solving a Fluid-Structure Interaction (FSI) problem. The FSI problem is solved with a partitioned approach, i.e. the flow and structural problem are solved in separate software codes and the required boundary conditions are exchanged between these solvers in every coupling iteration. The flow and structural solver are strongly-coupled, meaning that there are multiple coupling iterations per time step. The time step is the same for both flow and structural solver and is set to $5 \mathrm{e}-05 \mathrm{~s}$.

The flow side of the FSI problem is handled by ANSYS ${ }^{\circledR}$ Fluent ${ }^{\circledR}$ 17.1. The case is defined as stated in Section 2.3.1, except that the operating density $\rho_{\text {ref }}$ is now set explicitly set to zero. This is necessary because the hydrostatic pressure drop related to $\rho_{\text {ref }}$ is not taken into account in the pressure profile which is communicated to the structural solver. As such, a physically accurate load on the tube wall requires to set this reference density $\rho_{\text {ref }}$ to zero.

The structural part of the coupled problem is solved in the commercial software package Dassault Systèmes ${ }^{\circledR}$ Abaqus ${ }^{\circledR} 6.14$ which is a finite-element computational structural mechanics (CSM) code. The tube wall is modelled with linear hexahedral elements. Since the deformation of the wall is small (see Section 5), using a quadratic element would unnecessarily increase the computational time without meaningful accuracy gain. The reduced-integration scheme is applied since this considerably lowers the number of integration points per element and thus the computational effort, while offering good accuracy in typical bending problems. The tube is constrained such that it cannot move or deform at the inlet or outlet of the U-bend. As a result, a clamped-clamped configuration is modelled. The mesh contains 8000 elements and is shown in Figure 3.

A Dirichlet-Neumann decomposition is imposed at the fluid-structure interface, meaning that the pressure and shear stress are interpolated from the flow solver to the structural solver and the interface displacement is interpolated from

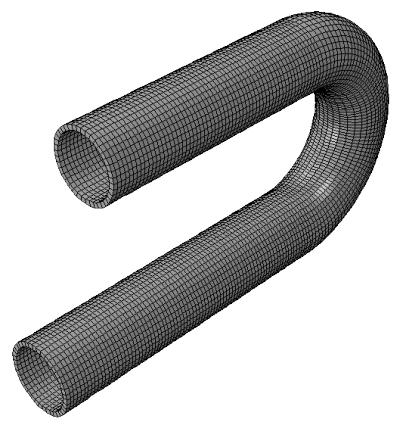

Figure 3: View of the mesh used in the structural solver. 
the structural solver to the flow solver. The equilibrium at the fluid-structure interface is enforced with the interface quasi-Newton algorithm with an approximation for the inverse of the Jacobian from a least-squares model (IQN-ILS) (Degroote et al. (2009)). This method is implemented in the in-house coupling code Tango, which handles the communication between the flow solver and the structural solver. The code Tango has been programmed in $\mathrm{C}++$ and has been validated for a number of different applications (Annerel et al. (2014); De Ridder et al. $(2015,2017)$ ), including the simulation of sloshing in a tank where the fluid subproblem was modelled with the VOF technique (Degroote et al. (2010)) similar to the method applied in the flow problem presented in this paper. As both solvers are commercial codes and therefore are black-box solvers, the Jacobian of the FSI-system is unknown. Therefore, an approximation of the inverse of the Jacobian is constructed, based on the residuals and displacements of previous coupling iterations. The FSI solver proceeds to the next time step when the $L 2$-norm of the interface displacement is lower than $1 \mathrm{e}-09 \mathrm{~m}$ and the $L 2$-norm of the pressure on the interface is lower than $1 \mathrm{~Pa}$. At this point, it should be noted that these residuals are not normalized with the number of faces (36000 on the fluid side and 8040 on the solid side) on the tube wall, which is why these residuals are acceptable. The maximal number of coupling iterations per time step is set to 15 . Upon reaching this number, the coupling code ends the current iterative procedure and moves to the next time step. In that case, the convergence criteria listed above are not met. However, this only occurs in the first time steps of the coupled simulation, the output of which is not reported.

Although a common practice in partitioned FSI simulations in single-phase flow applications (Degroote et al. (2009)), the re-use of modes obtained from previous time steps is detrimental for the stability of the method. This is due to the fact that small air bubbles or, equivalently, small low- $\alpha_{w}$ zones embedded in the liquid create a local pressure maximum, as can be seen in Figure 4. This pressure maximum is reflected in the modes of the residual matrix used for the approximation of the inverse of the Jacobian. In a following time step, the air bubble has moved slightly more downstream, therefore some modes of an earlier time step containing these local pressure maxima do not represent accurately the pressure profile in the current time step. This possibly causes a numerical instability of the problem when modes are reused, which is why the reuse of modes from previous time steps is not applied in this research.

\section{Mesh convergence study in the fluid problem}

Before describing the physical aspects of the flow through the U-bend, a mesh convergence study is presented. In Section 3.1, a universal refinement is performed, both along the centerline of the tube (referred to as the "axial" direction) and in the cross-section of the tube. The focus will be on the axial force profile, which will be of major importance in the subsequent paragraphs. In Section 3.2, the $y^{+}$-values obtained along the tube wall are discussed in more 


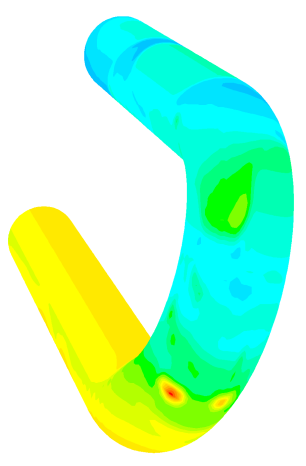

(a)

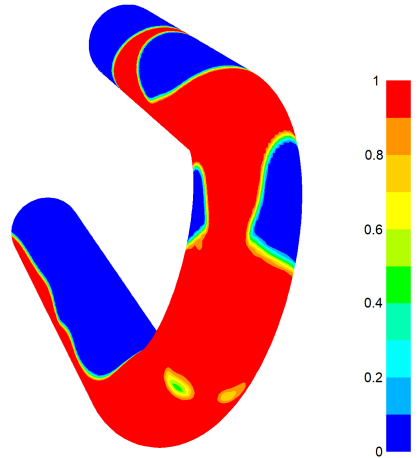

(b)

Figure 4: Contour plot of (a) pressure $p[\mathrm{~Pa}]$ and (b) $V O F_{w}[-]$ on the tube wall.

detail. A local mesh refinement is applied to investigate the boundary layer resolution.

\subsection{Global mesh refinement}

In this section, the numerical mesh is refined uniformly. All the cases to be discussed in the following mesh refinement study are listed in Table 1. In all cases, the variables $G, x$ and $\alpha_{w, \text { in }}$ equal $50 \mathrm{~kg} / \mathrm{m}^{2} \mathrm{~s}, 0.001$ and 0.3 , respectively. The cases of which the identification code start with an 'A' ("axial") are discussed in Section 3.1.1 and those with an 'R' ("radial") are discussed in Section 3.1.2. Preceding the conclusion of this section, it should be noted that cases $A 300$ and $R 30$ are identical and have the same specifications as case $I C 03$ defined in Table 3.

Table 1: List of cases used in the analysis of the mesh refinement study.

\begin{tabular}{|c|c|c|c|}
\hline Case & \#faces in cross-section [-] & \#axial divisions [-] & \#cells [-] \\
\hline A200 & 2,100 & 200 & 420,000 \\
A300 & 2,100 & 300 & 630,000 \\
A400 & 2,100 & 400 & 840,000 \\
\hline R20 & 960 & 300 & 288,000 \\
R30 & 2,100 & 300 & 630,000 \\
R40 & 3,680 & 300 & $1,104,000$ \\
\hline
\end{tabular}

\subsubsection{Axial refinement}

Three different axial resolutions are tested: $A 200, A 300$ and $A 400$. The three meshes only differ in the number of axial divisions and have the same 
resolution in each cross-section of the tube (2100 faces in each cross-section, with 120 divisions in the circumferential direction). For each case, the axial force as a function of time is shown in Figure 5. The discussion of the underlying physical phenomenon is postponed until Section 4.2. The force profiles have been translated in time such that a narrow force peak corresponds to time instant $0 \mathrm{~s}$ in all cases. The force profile is quasi-periodic and the period is about $0.15 \mathrm{~s}$ for all three cases; the relative difference in period between case $A 300$ and $A 400$ is $2.3 \%$. It is noteworthy that the peak height at time 0s increases when the mesh is refined. In contrast, the integral under the force peak at $t=0 \mathrm{~s}$ does not change significantly due to mesh refinement, as reported in Table 2. Finally, the force profile shape in between force peaks at $0 \mathrm{~s}$ and $0.15 \mathrm{~s}$ is very similar for all three cases, indicating that all meshes grasp a similar flow behavior inside the tube. The exact values of the force differ, because the precise location at which certain bubble dynamics occur is very prone to small (numerical) instabilities. At each point, the values of the axial force found in cases $A 300$ and $A 400$ differ at most $0.003 \mathrm{~N}$. Overall, the mesh containing 630,000 cells is deemed sufficiently accurate to model the flow inside the bend, at least for the prediction of the force on the bend wall.

Table 2: Period of the axial force profile and integral under the force peak at $t=0 \mathrm{~s}$.

\begin{tabular}{|c|c|c|}
\hline Case & Period $[\mathrm{s}]$ & Force integral $[\mathrm{N} . \mathrm{s}]$ \\
\hline A200 & 0.1481 & $3.24 \mathrm{e}-06$ \\
A300 & 0.1516 & $3.18 \mathrm{e}-06$ \\
A400 & 0.1483 & $3.29 \mathrm{e}-06$ \\
\hline R20 & 0.1489 & $3.46 \mathrm{e}-06$ \\
R30 & 0.1516 & $3.18 \mathrm{e}-06$ \\
R40 & 0.1533 & $3.30 \mathrm{e}-06$ \\
\hline
\end{tabular}

\subsubsection{Cross-sectional refinement}

Three different mesh resolutions are tested: $R 20, R 30$ and $R 40$. As in previous section, the focus will be on the axial force profile. For each case, the axial force as a function of time is shown in Figure 6. The results of this analysis are very similar to those discussed in Section 3.1.1: the period of the axial force hardly changes between the coarsest and the finest mesh. The relative difference between the periods found in cases $R 30$ and $R 40$ is $1.1 \%$. Also, the typical force profile shape is found for all three cases, but the exact value of the narrow force peak at time instant $0 \mathrm{~s}$ is uncertain and increases with the number of cells. At each point, the values of the axial force found in cases $R 30$ and $R 40$ differ at most $0.005 \mathrm{~N}$. In conclusion, the mesh containing 630,000 cells allows to adequately model the flow inside the bend and predict the force on the bend wall. 


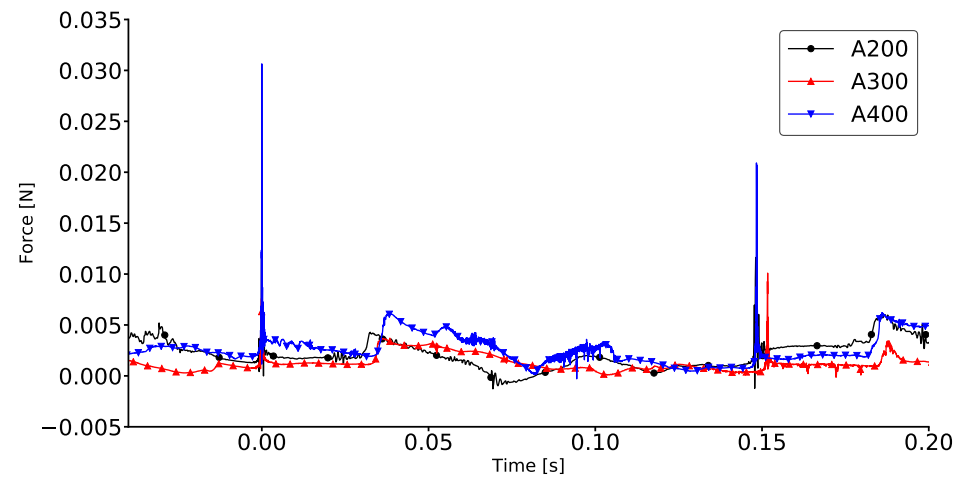

Figure 5: Force in the axial direction as a function of time for cases A200, A300 and A400.

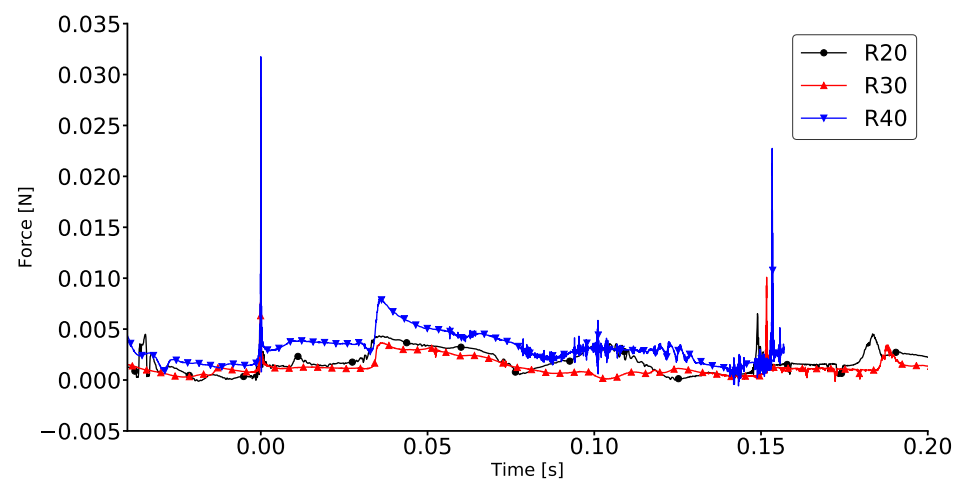

Figure 6: Force in the axial direction as a function of time for cases R20, R30 and R40. 


\subsection{Local mesh refinement}

Aside from the global mesh refinement study performed in Section 3.1, the resolution of the boundary layer close to the tube wall should be investigated. The $y^{+}$-values of the reference case IC03 (see Table 3 ) are all below 5 for the majority of calculated time steps. Only close to the time instant where a narrow force peak occurs, some $y^{+}$-values are found in the range of $5-8$. The reason is that the incompressible air flow has to move through a fine gap in between the large water layer and the tube wall, just prior to impact. The $y^{+}$-profile at this severe time instant is shown in Figure 8a. In an attempt to improve the $y^{+}$-resolution, the mesh was refined locally, as shown in Figure 7. The mesh resolution was only refined in the zone surrounding the point of liquid impact. The $y^{+}$-profile found with the refined numerical mesh during the next air entrapment nonetheless contains values above 5 . The $y^{+}$-profile is given in Figure 8b.

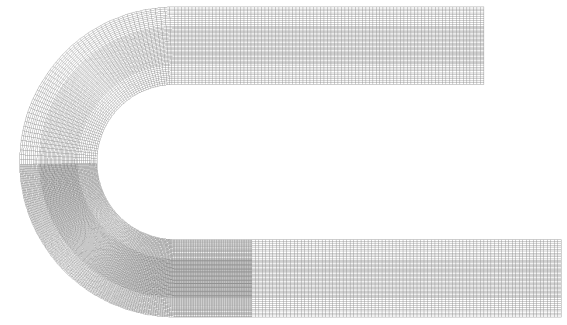

(a)

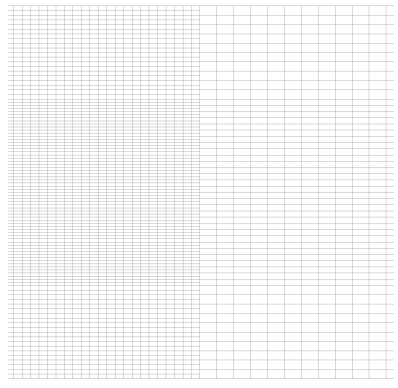

(b)

Figure 7: View of the refined mesh containing 1,688, 407 cells: (a) midplane view of the entire mesh and (b) zoom on the transition between the original and refined mesh zones

Presumably, this $y^{+}$-peak cannot be avoided within the limits of this numerical simulation due to the modelling of the phases as being incompressible. This is not a severe issue for the validity of the simulations for two reasons. Firstly, it should be noted that this $y^{+}$-peak does not persist for a long period of time: about $3 \mathrm{~ms}$ after the bubble formation, all $y^{+}$-values are again below 5. Secondly, it can be shown that the influence of the turbulence model and wall shear stress is limited, by comparing the axial force profile in Figure 14 to the axial force found for a laminar calculation, which is shown in Figure 9. Except for the peak value of the force, which was not reliably determined in the first place, the flow profiles are almost identical for the calculation applying the $k-\omega S S T$ model and the laminar model, respectively.

For the sake of completeness, the axial force profile obtained for the calculation with the refined mesh is given in Figure 10. This profile is close to the force signal obtained for the reference case, except for the value of the force peaks. It 


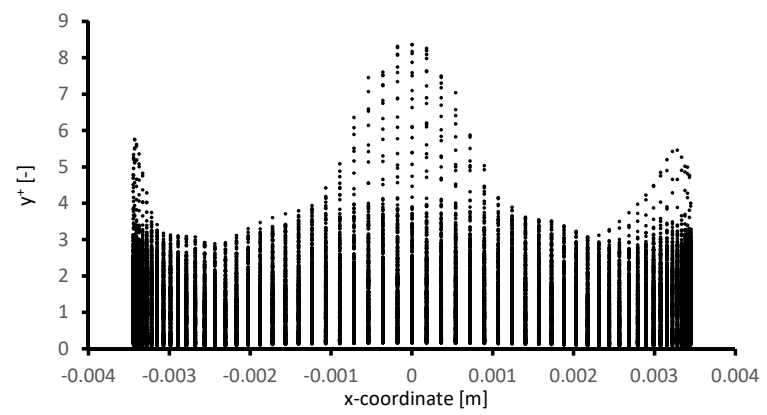

(a)

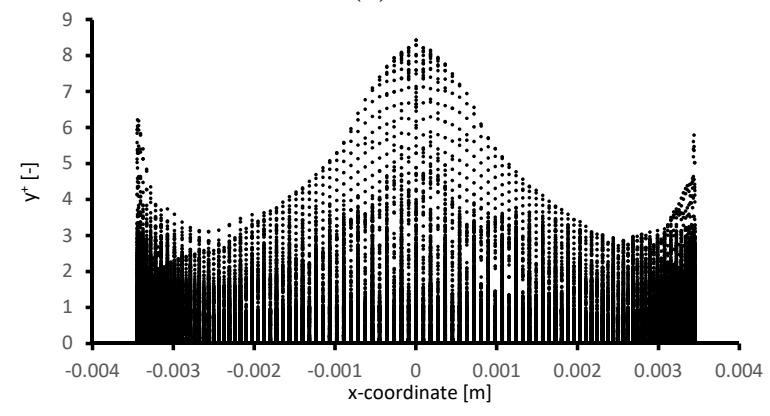

(b)

Figure 8: $y^{+}$-profile of all near-wall cells at the most severe time instant (bubble formation) for (a) the reference mesh and (b) the refined mesh

is not expected that further refinement would improve the convergence of the maximal force value, but the time integral under a narrow force peak is similar in both cases: about $3.2-3.9$ e-06 Ns in the reference case and 3.7 e-06 Ns for the refined mesh.

\section{Flow through a rigid tube}

In this paragraph, a CFD analysis is performed on a rigid tube. In Section 4.1, the characteristic flow profile is described, with the focus on the typical contour plots obtained for velocity, pressure and water volume fraction. The force data on the tube wall are presented in Section 4.2. Next, the effect of two liquid parameters is studied (Section 4.3). The influence of the inlet condition will be thoroughly described in Section 4.4 and it will be shown that the flow profile completely changes for high mass flow rates in Section 4.5. Finally, the effect of the operating density in incompressible flow calculations (Section 4.6) and the effect of the compressibility of the flow (Section 4.7) will be presented. In this broad CFD analysis, a number of different cases will be used, which are all summarized in Table 3. Four non-dimensional parameters are defined in this 


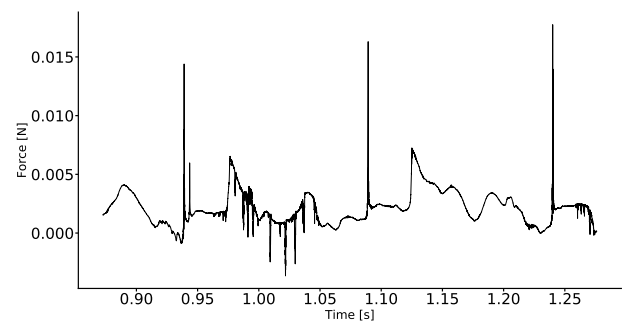

Figure 9: Axial force profile as a function of time for case $I C 03$ considering laminar flow.

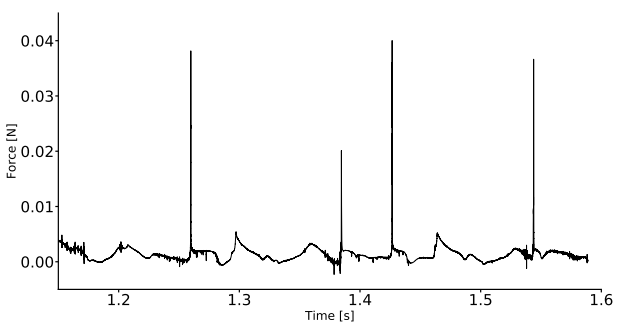

(a)

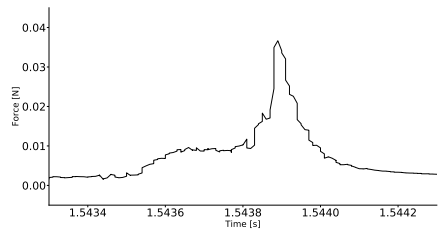

(b)

Figure 10: Force in the axial direction as a function of time for the refined mesh: (a) global view and (b) zoom on the narrow force peak around $t=1.543 \mathrm{~s}$

table: the Reynolds number $(R e)$, the Weber number $(W e)$, the capillary number $(\mathrm{Ca})$ and the Froude number $(\mathrm{Fr})$. Their definitions are given below (based on mixture properties):

$$
\begin{gathered}
R e=\frac{\rho_{m} U_{m} D}{\mu_{m}} \\
W e=\frac{\rho_{m} U_{m}^{2} D}{\sigma}
\end{gathered}
$$




$$
\begin{gathered}
C a=\frac{\mu_{m} U_{m}}{\sigma} \\
F r=\frac{U_{m}}{\sqrt{g D}}
\end{gathered}
$$

where $U_{m}$ is defined as the ratio of the mass flux $G$ and $\rho_{m}$. For the case $G 100 s$, these parameters are not calculated because the slug flow regime is not well represented by a single value of these parameters. This omission does not limit the discussion presented in the following sections.

The computational time of each case in Table 3 is similar and is about 135 hours on 6 nodes, each containing 2 processors of type Intel Xeon E5 2680v4 (resulting in 28 cores per node or 168 cores in total). During that time, about 1s of physical flow time was calculated. The cases will be identified further by using the code defined in the first column of this table.

Table 3: List of cases used in the CFD analysis. Columns 1 to 6 represent respectively the case identifier, the volume-of-fluid of water imposed at the inlet, the mass flux, the air mass fraction, the operating density and the number of cells in the computational mesh. In case the volume-of-fluid inlet profile is preceded by ' $\sim$ ', the inlet profile was taken from a fullydeveloped flow in a long tube, as discussed in Section 4.5 and therefore exhibits a more diffuse interface. In case $G 100 s$, a slug flow is modelled, where the air bubble takes up $50 \%$ of the surface area, followed by a period during which only water enters the U-bend. Columns 7 to 10 contain the non-dimensional parameter values.

\begin{tabular}{|c|c|c|c|c|c|c|c|c|c|}
\hline Case & $\alpha_{w, i n}[-]$ & $G\left[\mathrm{~kg} / \mathrm{m}^{2} \mathrm{~s}\right]$ & $x[-]$ & $\rho_{\text {ref }}\left[\mathrm{kg} / \mathrm{m}^{3}\right]$ & $\#$ cells $[-]$ & $R e[-]$ & $W e[-]$ & $C a[-]$ & $F r[-]$ \\
\hline IC01 & 0.1 & 50 & 0.001 & 124 & 630,000 & 2964 & 2.35 & 0.00079 & 1.90 \\
IC03 & 0.3 & 50 & 0.001 & 124 & 630,000 & 1103 & 0.79 & 0.00071 & 0.64 \\
IC05 & 0.5 & 50 & 0.001 & 124 & 630,000 & 678 & 0.47 & 0.00070 & 0.38 \\
IC07 & 0.7 & 50 & 0.001 & 124 & 630,000 & 489 & 0.34 & 0.00069 & 0.27 \\
G50 & $\sim 0.7$ & 50 & 0.001 & 124 & 630,000 & 489 & 0.34 & 0.00069 & 0.27 \\
G50o & $\sim 0.7$ & 50 & 0.001 & 0 & 630,000 & 489 & 0.34 & 0.00069 & 0.27 \\
G75 & $\sim 0.7$ & 75 & 0.001 & 124 & 630,000 & 734 & 0.76 & 0.00104 & 0.41 \\
G75o & $\sim 0.7$ & 75 & 0.001 & 0 & 630,000 & 734 & 0.76 & 0.00104 & 0.41 \\
G100 & $\sim 0.7$ & 100 & 0.001 & 124 & 630,000 & 978 & 1.35 & 0.00138 & 0.55 \\
G100o & $\sim 0.7$ & 100 & 0.001 & 0 & 630,000 & 978 & 1.35 & 0.00138 & 0.55 \\
G100s & $0.5 / 1$ & 98.5 & 0.0006 & 124 & 630,000 & - & - & - & - \\
M300 & 0.3 & 300 & 0.009 & 135 & $1,230,768$ & 6619 & 28.37 & 0.00429 & 3.83 \\
\hline
\end{tabular}

\subsection{Flow profile}

In order to get a first look at the characteristics of the flow with a low mass flux, case IC03 is considered in this section. With an inlet mass flux of $50 \mathrm{~kg} / \mathrm{m}^{2} \mathrm{~s}$, the flow inside the U-bend is characterized by flow reversal in the bend. Since a constant mass flux enters the domain through the inlet, a liquid layer build-up arises near the bottom of the bent part of the tube, until the cross-section is completely filled with water. The $\alpha_{w}$-profile on the symmetry plane just prior to this instant of bubble formation is shown in Figure 11a. Consequently, the continuous air layer is split into two parts and an air bubble is 
formed near the inner wall of the U-bend, as seen in Figure 11b. The flow reversal of the liquid due to gravity inside the U-bend is critical for this phenomenon to occur and is highlighted in Figure 12.

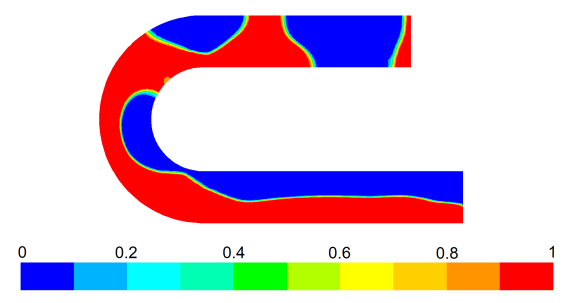

(a)

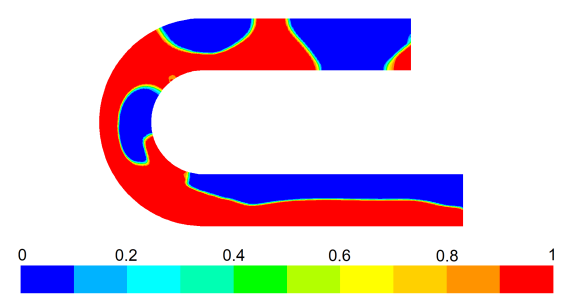

(b)

Figure 11: Contour graph of $\alpha_{w}$ [-] on the symmetry plane of the U-bend (a) prior to the onset of the air bubble in the bend, defined as time instant $T$ and (b) after bubble formation, at time instant $T+0.01 \mathrm{~s}$.

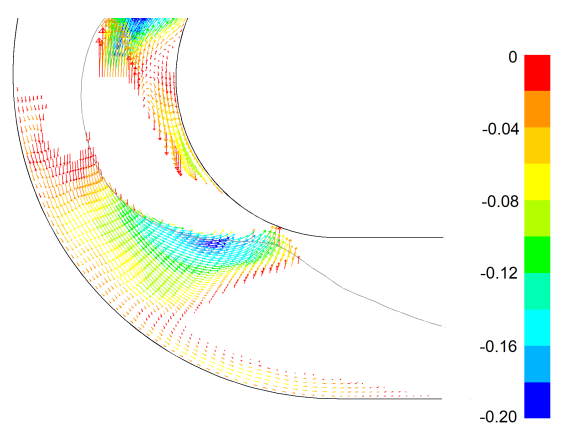

Figure 12: Vector graph on the U-bend symmetry plane showing the flow reversal inside the bend for case $I C 03$ at time instant $T$. The vectors are colored according to the value of the velocity component perpendicular to the inlet plane (expressed in $\mathrm{m} / \mathrm{s}$ ). Only vectors with a negative axial velocity component are shown. The black line denotes the tube wall, whereas the isosurface where $\alpha_{w}$ equals 0.5 is shown in grey. 
After the liquid layer impact on the wall and the related air bubble formation, the bubble moves along the U-bend. Initially, it stays attached to the inner wall of the bend, but close to the outlet pipe, the bubble migrates towards the outer wall due to buoyancy. This is shown in Figure 13.

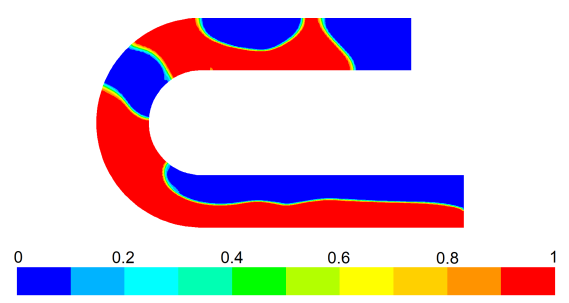

(a)

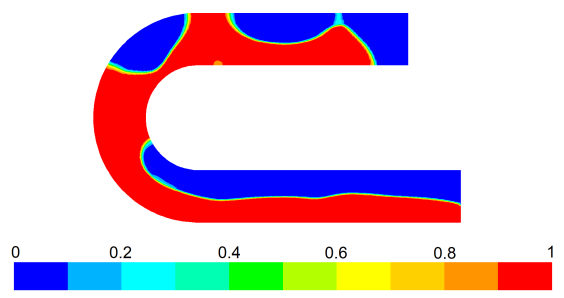

(b)

Figure 13: Contour plot of $\alpha_{w}[-]$ on the symmetry plane (a) at time instant $T+0.06 \mathrm{~s}$ and (b) at time instant $T+0.095 \mathrm{~s}$.

Wang et al. (2008) only published pictures of the water-air distribution found in the transparent U-bend section of their experimental set-up. Comparing the $\alpha_{w}$-profile found in the present simulations to their experimental results, it follows that the point of impact of the liquid layer is not entirely in agreement with the experiments with $x=0.001$. It is difficult to draw a conclusion from this discrepancy since Wang et al. (2008) do not describe the exact flow conditions at the inlet of the test section, but it appears that the incoming air layer is not continuous for $x=0.001$. Rather, liquid slugs seem to enter the U-bend, contrary to the stratified air-water profile used in the simulations. The characteristic speed and size of these slugs are not reported by Wang et al. (2008). It follows that reporting the values of $G$ and $x$ is not sufficient to replicate the exact experimentally observed phenomena in the geometry. Ideally for appropriate comparison between numerical and experimental investigations, the transient behaviour at in- and outlet should be reported.

\subsection{Axial force profile}

The previously described flow behavior leads to a characteristic axial force profile. In this case, the axial force refers to the force in the horizontal direction, perpendicular to the inlet face. This force signal is plotted in Figure 14 for the 
reference case $I C 03$. The force profile is dominated by a very sharp peak, e.g. at time instant 1.09s. Moreover, the time interval in between two consecutive peaks is approximately constant and equal to about $0.15 \mathrm{~s}$. The peak corresponds to the formation of the air bubble in the U-bend, coinciding with a liquid impact on the inner wall of the bend. This impact moment is preceded by a high speed air flow, squeezed in a small gap between the rising water levels and the rigid tube wall. This creates a low-pressure zone on the inner bend wall, provoking the force peak. It is clear that the maximal value is not exactly determined: the height of the narrow force peak differs from one period to the next. This does not mean that the peak is not fully resolved; figure 14b shows that the peak around $1.39 \mathrm{~s}$ contains several data points; the same holds for the other force peaks.

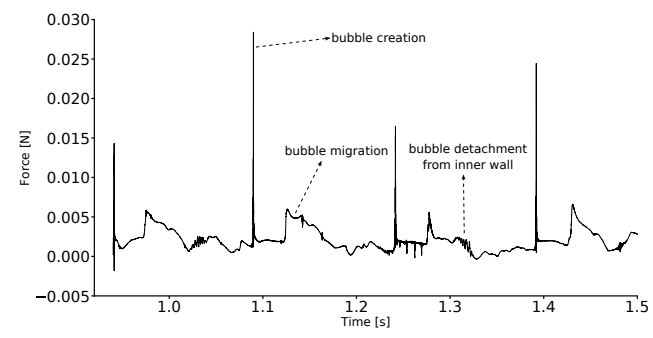

(a)

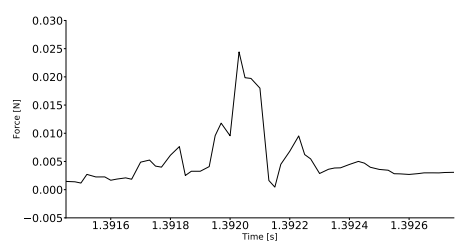

(b)

Figure 14: Force in the axial direction as a function of time for case IC03: (a) global view and (b) zoom on the narrow force peak around $t=1.392 \mathrm{~s}$.

The air downstream of the liquid build-up is now separated from the air layer at the inlet of the domain and moves further up the bend due to its initial flow speed and due to buoyancy. In the force profile, a more continuous zone of slightly elevated pressure is encountered. This corresponds to the time period required for the air bubble to migrate from the inner part of the tube to the outer part due to buoyancy. During the gas transport across the cross-section, liquid gets displaced and pushes against the outer tube wall. Yet, there is no impact of liquid nor a strong pressure build-up associated to this motion, such that the pressure elevation is moderate. Additionally, changing from period to period, 
high-frequency oscillations appear in the axial force profile, e.g. at $t=1.3 \mathrm{~s}$. This is typically the result of the oscillation of the gas-liquid interface, which has a clear contribution on the pressure and thus the force. At this particular time instant, the bubble has almost finished moving towards the outer part of the tube, but it remains attached by a narrow gas strip to the inner wall of the bend. When the air bubble detaches, the interface quickly bounces back to form a spherical bubble shape and it experiences some pulsations which gradually damp out. This oscillation is stronger due to the incompressible nature of the fluid. In compressible flow, the pulsations would not be transported throughout the entire domain.

\subsection{Influence of liquid viscosity and wall wetting angle}

The influence of the liquid viscosity and wall wetting on the flow profile is investigated in this section. All simulations described in this paragraph have been performed on the reference mesh with the inlet conditions of case IC03.

The liquid viscosity has a limited effect on the flow profile. The $\alpha_{w}$-profile is shown for a time instant just after bubble formation and for liquid viscosity equal to $\mu_{w}=0.001 \mathrm{~kg} / \mathrm{ms}$ (Figure 15a) and $\mu_{w}=0.005 \mathrm{~kg} / \mathrm{ms}$ (Figure $15 \mathrm{~b}$ ). The liquid layer prior to the bend entrance looks more unstable for the case with the higher viscosity. This observation is in accordance with the work described by Tzotzi et al. (2011), who found that an increasing liquid viscosity facilitates the onset of slug flow at low gas velocities. The effect of liquid viscosity on the flow profile is limited, as is to be expected in the range of low capillary numbers (respectively 0.00071 and 0.00357 ). However, the slightly changed behaviour in the inlet tube does not seem to affect the phenomena of interest in this research: the bubble formation mechanism is not altered due to the viscosity change and therefore the time period of the force signal is not dependent on the liquid viscosity (not shown). This is expected if the liquid build-up at the bend entrance is only dependent on the amount of liquid mass being transported to it. Finally, the bubble closest to the outlet is larger in Figure 15a than in Figure 15b, but this is due to the fact the hydrostatic pressure at the outlet is not completely in equilibrium with the boundary condition, allowing some acceleration of the gas bubble on the one hand and some flow reversal of air on the other hand (as mentioned in Section 2.3.1).

The contact angle between the air-water interface is defined as a boundary condition for $\alpha_{w}$ at the wall. This contact angle is a quantitative measure of the wall wettability: large contact angles correspond to hydrophobic walls and small contact angles to hydrophilic walls. Two cases are defined, where the contact angle equals $90^{\circ}$ and $114^{\circ}$, respectively. The other fluid parameters and boundary conditions are the same for both cases. The contour plot of $\alpha_{w}$ in the two distinct cases is shown in Figure 16. It is clear that the liquid-air interface has a different shape close to the wall, which is a direct result from the different wall boundary condition for $\alpha_{w}$. The contact angle could affect the periodicity of the force on the bend if the amount of air in a single bubble would vary significantly with the value of the contact angle. However, nothing indicates that the bubble formation is in any way affected by this, unless by a 


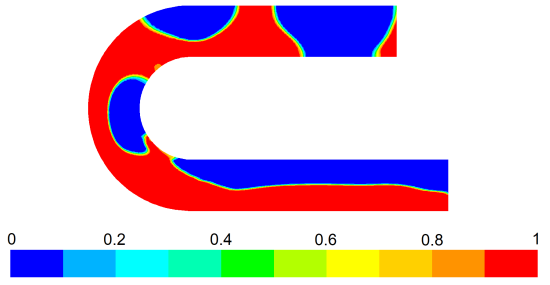

(a)

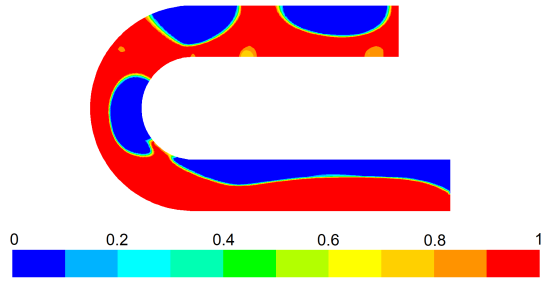

(b)

Figure 15: Contour plot of $\alpha_{w}[-]$ on the midplane at similar time instants for following values of liquid viscosity: (a) $\mu_{w}=0.001 \mathrm{~kg} / \mathrm{ms}$ and (b) $\mu_{w}=0.005 \mathrm{~kg} / \mathrm{ms}$

slight shift of the point of impact of the liquid layer. The phenomenon analyzed in this work occurs independently of the type of coating applied on the inside of the tube wall.

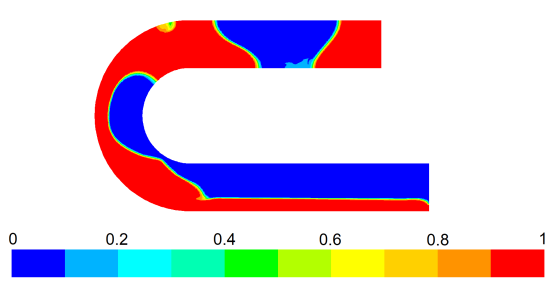

(a)

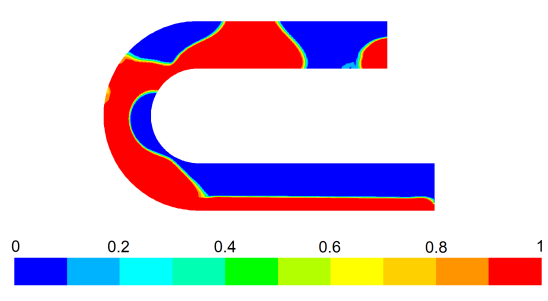

(b)

Figure 16: Contour plot of $\alpha_{w}[-]$ on the midplane at similar time instants for following values of the contact angle between the gas-liquid interface and the tube wall: (a) $90^{\circ}$ and (b) $114^{\circ}$.

\subsection{Influence of the inlet condition: applying different water heights}

The inlet condition is not completely determined by the definition of a mass flux $G$ and air quality $x$. Because the phases are assumed incompressible, these two parameters define the product of phase area and phase velocity at the inlet: $A_{w, i n} U_{w, i n}$ and $A_{a, i n} U_{a, i n}$. Also, the sum of the areas taken up by both phases equals the tube cross-section: $A_{w, i n}+A_{a, i n}=A_{i n}$. Equivalently, one can state in terms of $\alpha_{w, i n}=A_{w, i n} / A_{i n}$ that the following products are fixed by defining $G$ and $x$ : $\alpha_{w, i n} U_{w, i n}$ and $\left(1-\alpha_{w, i n}\right) U_{a, i n}$. This means that either $U_{w, i n}, U_{a, i n}$ or $\alpha_{w, i n}$ should be defined independently in order to set the inlet condition unambiguously. This is an important issue, since the values for none of these parameters in the experiment by Wang et al. (2008) are known. Therefore, it is investigated whether changing the inlet condition for fixed values of $G=50 \mathrm{~kg} / \mathrm{m}^{2} \mathrm{~s}$ and $x=0.001$ has an important effect on the flow. Only 
stratified flow inlets are considered in this section. By contrast, a slug flow inlet will be investigated in Section 4.5.2.

The water level (thus the area taken up by the water, $A_{w, i n}$ ) is varied, such that the void fraction of water at the inlet equals $0.1,0.3,0.5$ and 0.7 , respectively. This corresponds to the cases defined with identifiers $I C 01, I C 03$, $I C 05$ and $I C 07$ in Table 3, respectively.

From the force profile depicted in Figure 17 it is found that there are a number of qualitative similarities between the different cases. Firstly, the force profiles for different cases are qualitatively the same. All cases exhibit a narrow peak at time 0s because the time axis was translated for every case separately so as to make an instant of liquid impact coincide with time 0s. Secondly, the broader pressure plateau occurring due to the bubble migration in the bend occurs as well for every case. Quantitatively, however, the period between two adjacent pressure peaks changes significantly. For cases $I C 05$ and $I C 07$, the time interval between two bubble initiations equals $0.12 \mathrm{~s}$ and $0.11 \mathrm{~s}$, respectively. For case $I C 01$, the period increases up to a value of $0.31 \mathrm{~s}$, about double of the original case with $V O F_{a, i n}=0.7$. The reason is that the narrow pressure peak only occurs when the liquid layer near the inlet of the bend has built up sufficiently to fill the entire cross-section with water at a point close to the inlet of the bend. As such, when the inlet $\alpha_{w}$ is smaller, the time required to build up the layer is larger as well, even though the incoming mass flux and thus also the volumetric flow rate remain the same. The fact that a slightly larger period was obtained for case $I C 07$ with respect to case $I C 05$ could indicate that less flow reversal occurs when the volume taken up by the air is small. Finally, the force profile for case IC 01 exhibits more intermediate peaks (peaks not related to bubble formation in the bend) than other cases. It is found that the formation of a large air bubble such as at time 0 s is immediately followed by the formation of a small bubble when the air layer at the inlet is thick. Both bubbles eventually coalesce inside the bend. This explains the force peaks in the period $0.10-0.20 \mathrm{~s}$, as the secondary bubble formation and coalescence are linked to sudden changes in the acceleration of the liquid.

In Section 4.5 it will be investigated how the mass flux affects the flow inside the U-bend. The observation that there is a clear effect of $\alpha_{w, i n}$, means that the definition of an appropriate inlet condition is crucial for the subsequent analysis, in order to be sure that the observed differences between the different mass flow rates are not due to the arbitrarily chosen inlet condition. This issue will be addressed by considering a fully-developed flow in Section 4.5. However, it should be noted that the results presented in Section 4.4 are still valuable, since non-equilibrium flows also occur in some heat exchanger geometries (De Kerpel et al. (2012)). It is therefore useful to see how the $\alpha_{w}$-profile affects the vibrations. Inside a heat exchanger with limited dimensions, other time frequencies could be dominant in the force profile, compared to those derived from simulations or experiments during which the flow was allowed to fully develop. 


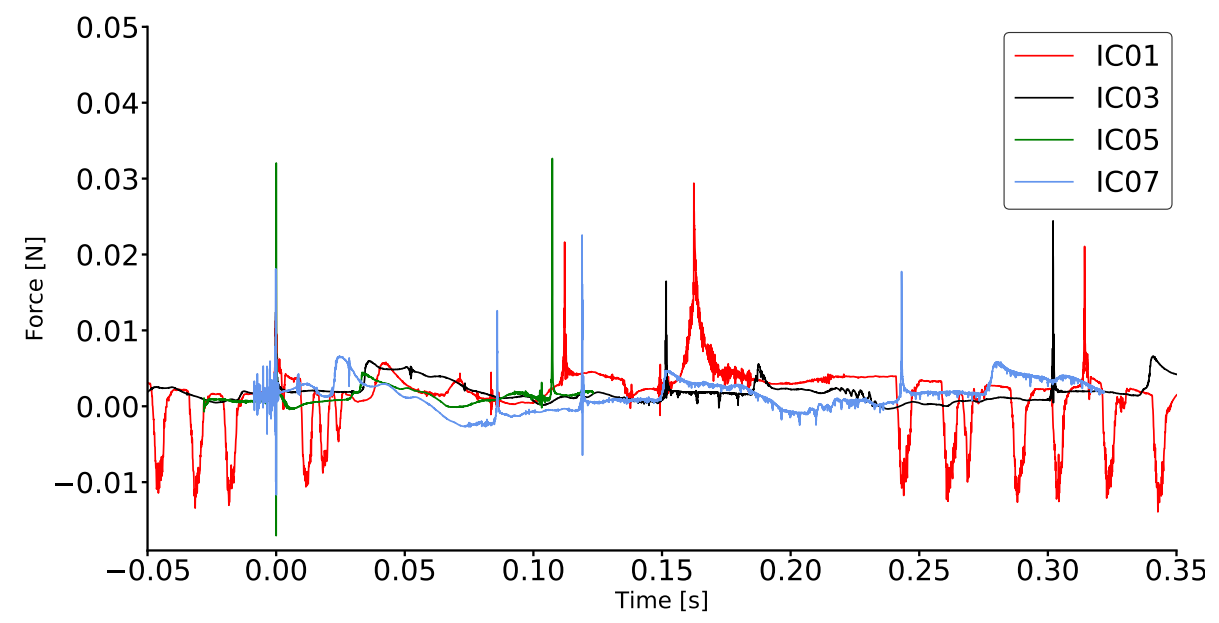

Figure 17: Force in the axial direction as a function of time for different cases. The time scale is chosen such that a bubble is formed at time instant $t=0 \mathrm{~s}$ in all cases.

\subsection{Influence of the mass flow rate}

\subsubsection{Stratified inlet condition}

The precise knowledge of the inlet condition is necessary to get a good match with the experiments. This leads to two issues. Firstly, the exact flow distribution applied by Wang et al. (2008) is not reported and is therefore unknown to the authors of this article. Secondly, the inlet of the U-bend should only be a function of the imposed mass flux, not of an arbitrarily set phase distribution. In order to address these issues, the flow through a straight, horizontal pipe of $200 D$ long is simulated. The mesh applied in the long inlet tube was described in Section 2.1. At the inlet of the pipe, the same inlet profile is set as in the original case: air takes up $70 \%$ of the area of the tube. Three different mass fluxes are calculated: $50 \mathrm{~kg} / \mathrm{m}^{2} \mathrm{~s}, 75 \mathrm{~kg} / \mathrm{m}^{2} \mathrm{~s}$ and $100 \mathrm{~kg} / \mathrm{m}^{2} \mathrm{~s}$. After the flow is allowed to develop, the $\alpha_{w^{-}}$and $U$-contours in a cross-section of the tube located at $0.5 \mathrm{~m}$ and $0.7 \mathrm{~m}$ from the inlet respectively, are compared. It was found that for the two lowest mass fluxes, the flow was fully developed. To show this, the $\alpha_{w^{-}}$and $U$-contours at $0.5 \mathrm{~m}$ from the inlet and the absolute error compared to the profile at $0.7 \mathrm{~m}$ from the inlet are shown in Figure 18 for $G=75 \mathrm{~kg} / \mathrm{m}^{2} \mathrm{~s}$; similar plots can be made for $G=50 \mathrm{~kg} / \mathrm{m}^{2} \mathrm{~s}$. For the highest mass flux, $G=100 \mathrm{~kg} / \mathrm{m}^{2} \mathrm{~s}$, slug flow develops, which will be discussed in Section 4.5.2.

The profiles for both $\alpha_{w}$ and $U$ found at $z=0.5 \mathrm{~m}$ are subsequently imposed at the inlet of the U-bend with the mesh containing 630,000 cells. This leads to the definition of the three cases in Table 3: G50, G75 and G100. In these three cases, gravity is dominant over the inertia of the liquid. Therefore, the previously described phenomenon of flow reversal followed by air bubble generation in the U-bend occurs in all of these calculations. The difference between the 


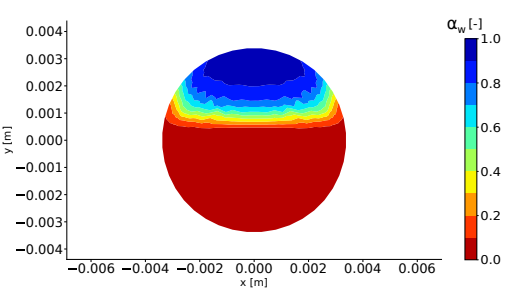

(a)

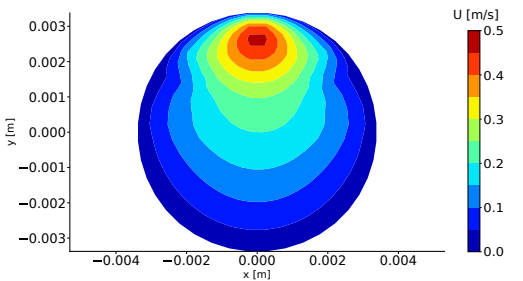

(c)

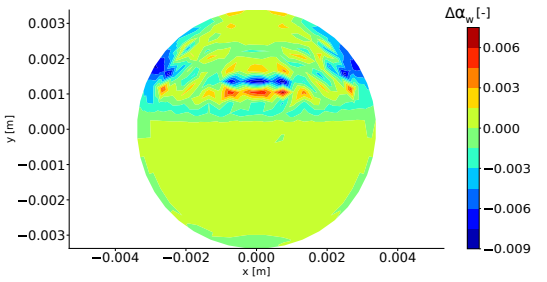

(b)

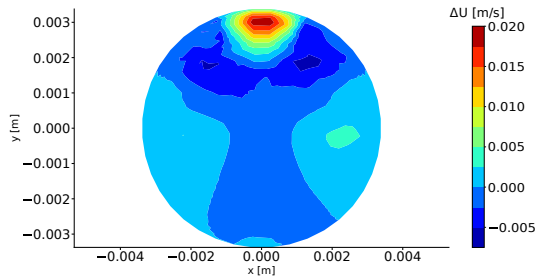

(d)

Figure 18: Contour graphs after $59 \mathrm{~s}$ of flow time for $G=75 \mathrm{~kg} / \mathrm{m}^{2} \mathrm{~s}$ and $x=0.001$ of the following profiles: (a) $\alpha_{w}$ at $0.5 \mathrm{~m}$ from the inlet, (b) $\alpha_{w}$ at $0.5 \mathrm{~m}$ from the inlet minus $\alpha_{w}$ at $0.7 \mathrm{~m}$ from the inlet, (c) $U$ at $0.5 \mathrm{~m}$ from the inlet and (d) $U$ at $0.5 \mathrm{~m}$ from the inlet minus $U$ at $0.7 \mathrm{~m}$ from the inlet. 
cases is the value of the period of the axial force signal and thus of the vibration occurring in the bend. The axial force signals for the three cases are shown in Figure 19. It is found that the period of the axial force equals, respectively: $0.14 \mathrm{~s}$ for $G=50 \mathrm{~kg} / \mathrm{m}^{2} \mathrm{~s}, 0.10 \mathrm{~s}$ for $G=75 \mathrm{~kg} / \mathrm{m}^{2} \mathrm{~s}$ and $0.07 \mathrm{~s}$ for $G=100 \mathrm{~kg} / \mathrm{m}^{2} \mathrm{~s}$. In conclusion, the period is inversely proportional to the incoming mass flux, which is logical if the dominant phenomenon - the liquid impact on the inner wall - is the same and requires a certain fixed amount of liquid present in the inlet pipe of the bend. Doubling the mass flow rate then halves the time required to achieve this amount of water and therefore doubles the frequency of occurrence of the axial force peak.

Finally, it should be noted that the foregoing analysis is only correct when gravity is dominant over the inertia forces in the bend. When the mass flux is superior to the values described before, e.g. $300 \mathrm{~kg} / \mathrm{m}^{2} \mathrm{~s}$, the inertia of the liquid will prevent flow reversal from happening in the bend, as can be observed in Figure 20, which depicts the $\alpha_{w}$-profile in the midplane section obtained for case $M 300$. For case $M 300$, the inlet and outlet were chosen at $10 D$ and $9 D$ from the bent part, respectively. The liquid layer just moves around the outer wall of the bend and enters the outlet tube on top of the air layer. There is no impact of liquid on the tube wall in such case and therefore the U-bend will not vibrate (although the force required to turn the mixture increases). The liquid will naturally drop down further downstream due to gravity, but this does not occur in the midplane of the domain. As the high Froude number (3.83) indicates, inertia is dominant over gravity in this regime. It should be noted that the Froude number in case $I C 01$ is 1.9 , so the Froude number limit between the gravity-dominant and inertia-dominant regime is between 1.9 and 3.8.

\subsubsection{Slug flow inlet}

General flow regime maps indicate that a stratified profile is obtained for given conditions in Section 4.4. However, Lin and Hanratty (1986) stated that changing the tube diameter severely alters the boundaries between flow regimes, specifically the onset of slug flow occurs at significantly lower gas flow rates for small tube diameters. Although the authors have not found flow regime maps adapted to a tube diameter of $0.0069 \mathrm{~m}$, it is certainly possible that slug flow develops for $G=100 \mathrm{~kg} / \mathrm{m}^{2} \mathrm{~s}$ and $x=0.001$. When calculating the flow in a long slender tube with $D=0.0069 \mathrm{~m}$, a slug flow indeed develops, as can be seen in Figures 21 and 22 . The air bubble velocity is extracted from the time history of this calculation and found to be equal to $0.205 \mathrm{~m} / \mathrm{s}$. The period between bubbles is measured to be $0.711 \mathrm{~s}$. The length of an air bubble is about $0.107 \mathrm{~m}$.

In order to obtain a continuous inlet condition, the liquid and air velocity are set to the bubble velocity $0.205 \mathrm{~m} / \mathrm{s}$. This means that, without modelling slip between the phases and without changing the velocity over time, the mass flux is not exactly equal to $G=100 \mathrm{~kg} / \mathrm{m}^{2} \mathrm{~s}$ and the air quality is not equal to 0.001 , as already noted for case G100s in Table 3. The discrepancy is due to the fact that in the fully-developed flow, the air and liquid velocity are not constant, nor is the velocity in the liquid layer under the air bubble necessarily the same as the liquid velocity between consecutive air bubbles. The obtained values, 


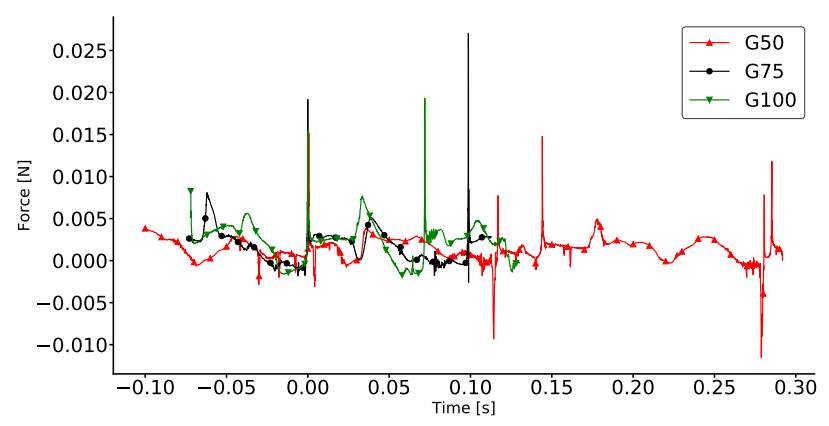

Figure 19: Axial force as a function of time for cases $G 50, G 75$ and $G 100$. The force signals are translated in time such that the time instant 0 corresponds to a point in time when a bubble is generated in the U-bend.
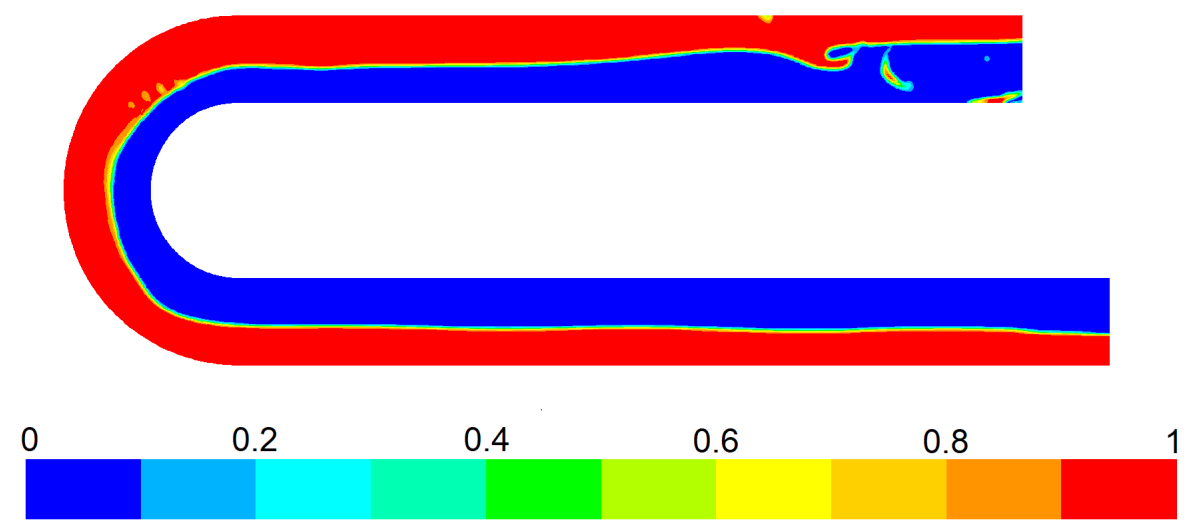

Figure 20: Contour plot of $\alpha_{w}[-]$ in the midplane section at a single time instant for an imposed mass flux of $300 \mathrm{~kg} / \mathrm{ms}$. Air takes up $70 \%$ of the cross-sectional area at the inlet.

however, are close to the original settings: $G=98.5 \mathrm{~kg} / \mathrm{m}^{2} \mathrm{~s}$ and $x=0.0006$. At the inlet of the U-bend, the inlet profile is dependent on time. During the first 0.463 s (corresponding to the air bubble length with given bubble velocity), an air bubble enters the domain. $50 \%$ of the tube's cross-section is then filled with air $\left(\alpha_{w}=0.5\right)$. During the remaining $0.248 s$ of the period, only liquid enters the bend $\left(\alpha_{w}=1\right)$.

The force profile obtained for the flow with the slug inlet condition is shown in Figure 23. Similar to the cases with stratified flow, the signal is periodic. The period of the signal is $0.067 \mathrm{~s}$, which is close to the period obtained for the stratified inlet profile in Section 4.5.1. This slight discrepancy is possibly due to the minor difference in $G$ and $x$, as indicated before. Each period contains an intermediate, smaller force peak, e.g. at time 0.505s in Figure 23b. This corresponds to the detachment of the air bubble from the inner wall and the 

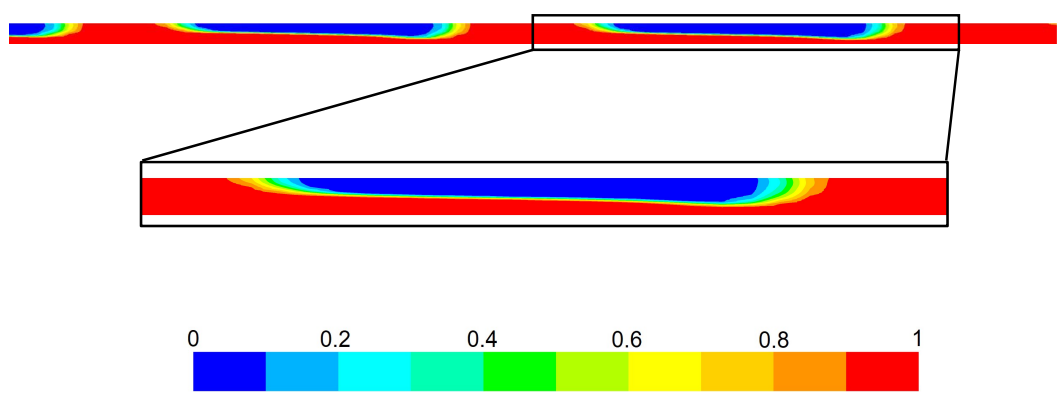

Figure 21: Contour graph of $\alpha_{w}$ [-] in the vertical symmetry plane of the long, slender tube with $G=100 \mathrm{~kg} / \mathrm{m}^{2}$ s and $x=0.001$ after 40 s of physical flow time. Only the last $50 D$ of the tube are shown.

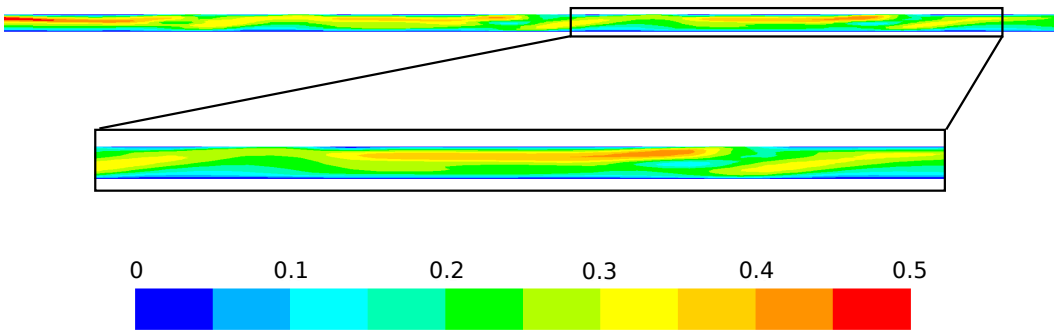

Figure 22: Contour graph of $U[\mathrm{~m} / \mathrm{s}]$ in the vertical symmetry plane of the long, slender tube with $G=100 \mathrm{~kg} / \mathrm{m}^{2} \mathrm{~s}$ and $x=0.001$ after 40 s of physical flow time. Only the last $50 D$ of the tube are shown. 
coincident reattachment to the outside wall, which was encountered before. It is remarkable that, if the air bubble is long enough to allow sufficient flow reversal in the bend, the same phenomenon of liquid impact and air entrapment occurs. Obviously, during the final 0.248 s of each period only water enters the bend. This has an effect on the force profile: no impact forces occur when the bent part is completely filled with water. The axial force exhibits a near-constant, strictly positive value when the bend does not contain air. Consequently, the force signal is only periodic when an air slug is present in the bend.

\subsection{Influence of the operating density}

In the foregoing analysis, the pressure at the outlet was fixed to a pressure field corresponding to the hydrostatic pressure for a fluid with a reference density $\rho_{\text {ref }}$ equal to $124 \mathrm{~kg} / \mathrm{m}^{3}$ (this is the volume-averaged density of the air-water mixture at the start of the simulations). As a result, the pressures that are reported by the CFD code are determined relative to this hydrostatic pressure drop and therefore the force, calculated as a surface integral from this pressure field, is as well. The definition of a non-zero operating density therefore has a direct effect on the reported force in the vertical direction since the corresponding hydrostatic pressure field was substracted from the actual force, but it does not directly affect the force in the axial direction. However, varying the operating density changes the outlet condition, which could therefore change the flow profile inside the bend. It should therefore be verified that the outlet condition does not affect the flow profile inside the bend. This is done by comparing the periods of the vibration in the cases $G 100$ (with $\rho_{\text {ref }}$ equal to $124 \mathrm{~kg} / \mathrm{m}^{3}$ ) and $G 100 o$ (with $\rho_{\text {ref }}$ equal to $0 \mathrm{~kg} / \mathrm{m}^{3}$ ). The force profiles of the CFD simulations are given in Figure 24. Two conclusions should be drawn from this figure. Firstly, the average force is significantly higher when the operating density is lower as a result of the force integration implemented in the flow solver; the force acting on the tube wall is calculated as follows:

$$
\vec{F}=\int_{\partial V} p_{c o r r} \vec{n} \mathrm{~d} A=\int_{\partial V} p \vec{n} \mathrm{~d} A-\int_{\partial V} \rho_{r e f} g h \vec{n} \mathrm{~d} A,
$$

where the tube wall is symbolized with $\partial V$, the normal vector on each wall face is defined as $\vec{n}$ and the surface is represented by $A$. Clearly, the definition of an operating density causes a negative shift in the force definition. Secondly, the flow mechanism - the creation of a large bubble inside the bend which subsequently migrates to the top of the return pipe - is unchanged, although the bubble migration does not cause an equally large local maximum in the force profile when the operating density is set to zero. It is important to note that the period of the force signal is not dependent on the value of the operating density, which confirms that the quantitative values for the axial force period given in Section 4.5.1 are independent of the exact outlet pressure distribution. This is a remarkable observation which is crucial for the validity of the reported periodicity, as the exact distribution at the pressure outlet is typically not known and is probably not constant over time (due to the changing water level height at the outlet). 


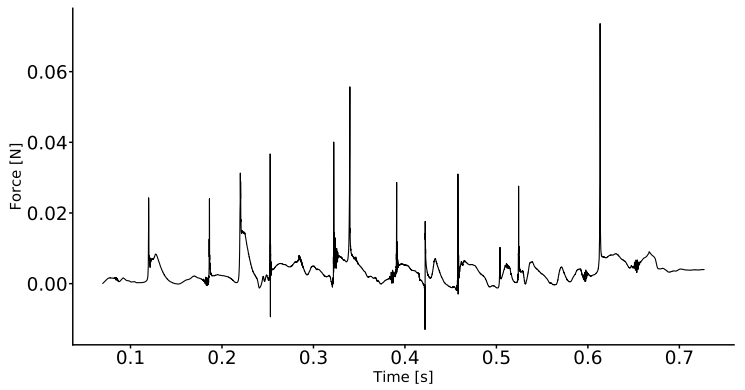

(a)

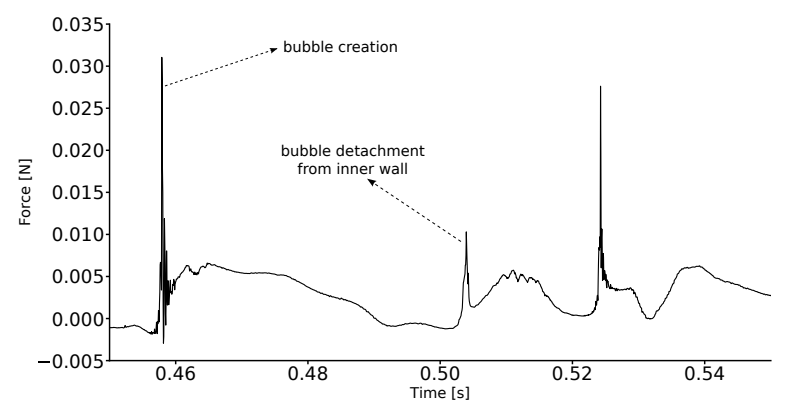

(b)

Figure 23: Axial force profile for case G100s with slug profile inlet (a) over a time period of $0.8 \mathrm{~s}$ and $(\mathrm{b})$ zoom on a time period of $0.1 \mathrm{~s}$.

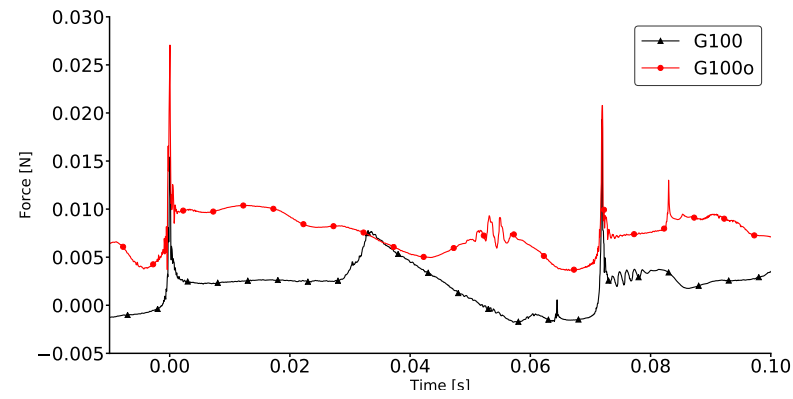

Figure 24: Comparison of the axial force profile for the cases $G 100$ (with $\rho_{\text {ref }}=124 \mathrm{~kg} / \mathrm{m}^{3}$ ) and $G 100 o$ (with $\rho_{\text {ref }}=0 \mathrm{~kg} / \mathrm{m}^{3}$ ). The horizontal axis was rescaled such that a force peak corresponds to time instant 0 for both cases. 


\subsection{Influence of the compressibility of the flow}

Water and air are only incompressible in theory. Generally speaking, the assumption of incompressible flow is acceptable when the pressure fluctuations are limited in amplitude. In the aforementioned simulations, the maximal observed pressure was about $1000 \mathrm{~Pa}$ occurring in a single cell of the mesh used in $I C 03$ at the moment of liquid impact on the wall. Outside of a range of 100 time steps before or after this instant, the pressure relative to the outlet is below 250Pa. Even though the elevated pressure level occurs locally and only for a small period of time, it could cause significant changes to the flow profile when considering compressible fluids. Therefore, three numerical simulations are performed in which air is considered an ideal gas. Water is either considered as a compressible fluid with a fixed bulk modulus $\left(K_{w}=2.2 \mathrm{e}+09 \mathrm{~Pa}\right)$ or as an incompressible liquid (with density equal to $1000 \mathrm{~kg} / \mathrm{m}^{3}$ ). The cases are summarized in Table 4 . The boundary conditions for pressure and velocity at the inlet and wall are the same as for case IC03. At the outlet, an absolute pressure $p_{\text {out }}$ has to be defined. The temperature is set to $300 \mathrm{~K}$ at both inlet and outlet (the latter as a backflow condition). The cell count of the numerical mesh equals 630,000 . In compressible flow cases, the energy equation has to be solved. Moreover, the $\alpha_{w}$-equation (Equation (4)) has to be modified as follows:

$$
1 / \rho_{w}\left[\frac{\partial\left(\alpha_{w} \rho_{w}\right)}{\partial t}+\nabla \cdot\left(\alpha_{w} \rho_{w} \vec{U}\right)\right]=0
$$

Table 4: List of cases with at least one compressible fluid. The case identifiers are listed in column 1. Column 2 and 3 indicate the models applied to air and water, respectively. The outlet pressure $p_{\text {out }}$ is defined in the last column.

\begin{tabular}{|c|c|c|c|}
\hline Case & Air & Water & $p_{\text {out }}[$ bar $]$ \\
\hline ACWC1 & ideal gas & compressible & 1 \\
ACWC5 & ideal gas & compressible & 5 \\
ACWI5 & ideal gas & incompressible & 5 \\
\hline
\end{tabular}

The axial force profiles found for the cases listed in Table 4 are shown in Figure 25. In compressible flow cases, a water impact on the tube wall causes a high-frequent force oscillation which is only slightly damped and therefore persists during the remainder of the force period. The value of this period remains nonetheless close to the incompressible flow case: about $0.14-0.15 \mathrm{~s}$ if $p_{\text {out }}$ equals $5 \mathrm{bar}$ and $0.17 \mathrm{~s}$ if $p_{\text {out }}$ equals $1 \mathrm{bar}$. The value of the outlet pressure $p_{\text {out }}$ has another significant effect: whereas case $A C W C 5$ exhibits an almost sinusoidal force fluctuation, the signal in case $A C W C 1$ is certainly not sinusoidal. Additionally, the time between two consecutive force maxima is different: about $0.012 \mathrm{~s}$ if $p_{\text {out }}$ is 1 bar and $0.005 \mathrm{~s}$ if $p_{\text {out }}$ equals 1 bar. The different dominant frequencies are highlighted in the real-valued Fourier spectra (Figure 26). It is clear from Figure 25b that the flow is not significantly altered when modelling water 
as an incompressible liquid; the axial force profiles obtained in cases $A C W C 5$ and $A C W I 5$ are comparable. Both simulations yield a slightly different average axial force (the difference is $4.7 \%$ ) and the time difference between force peaks is reported in Table 5 .

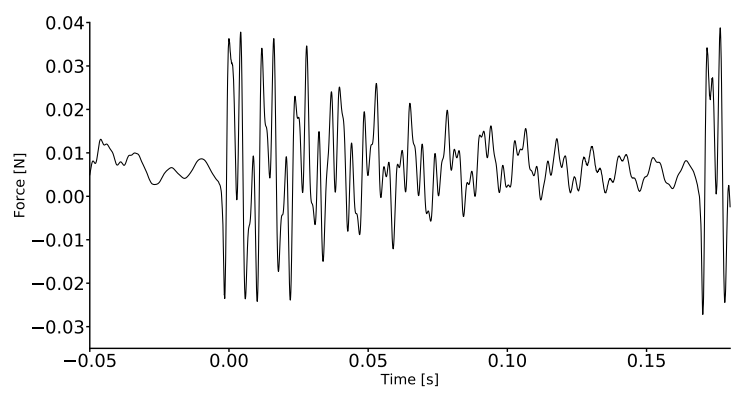

(a)

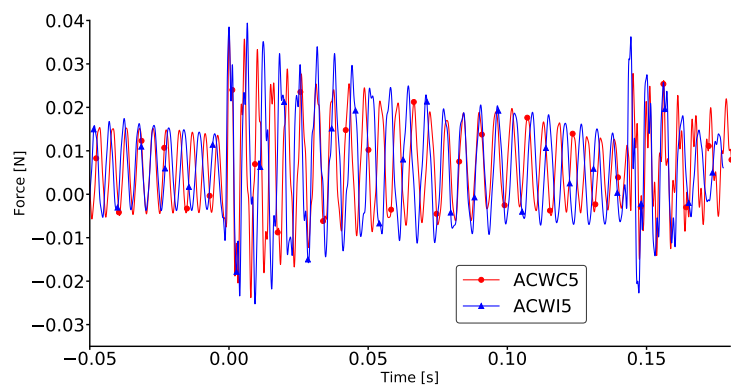

(b)

Figure 25: Axial force profile for (a) case $A C W C 1$ and (b) cases $A C W C 5$ and $A C W I 5$.

The occurrence of the high-frequent force oscillation is the main difference between the compressible and incompressible flow simulations. Its existence is related to the creation of a pressure wave at a time instant corresponding to the liquid impact on the bend wall. After its onset, the pressure wave moves back and forth through the numerical domain. A pressure wave is reflected at the outlet as a rarefaction wave because of the fixed pressure value imposed here. At the inlet, a pressure wave is reflected as a pressure wave as the pressure value is allowed to vary. Due to this configuration, it is hypothesized that the time difference between two consecutive force maxima, $\Delta t_{\max }$, corresponds to the time required for a wave to pass through the domain four times: twice as a pressure wave and twice as a rarefaction wave. As such, this time difference $\Delta t_{\max }$ is calculated as:

$$
\Delta t_{\max }=l / c
$$

where $l$ is the path length travelled by the wave and $c$ is the wave speed. 


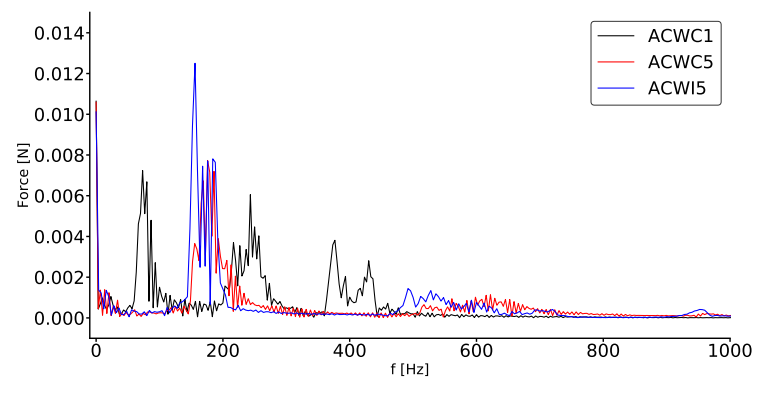

Figure 26: Real Discrete Fourier spectra of the axial force in $A C W C 1, A C W C 5$ and $A C W I 5$. The time intervals applied in the Fourier transform were $0.300 \mathrm{~s}, 0.296 \mathrm{~s}$ and $0.289 \mathrm{~s}$ long, respectively.

The determination of the wave speed $c$ is not straightforward, especially since animations of the pressure distribution indicate that the wave speed is greater in the largest part of the inlet tube (i.e. in the first part of the tube with length $4 D$ ) than in the rest of the domain. This discrepancy can be explained due to the different $\alpha_{w}$-profile: the flow is stratified in the inlet tube, whereas the part of the domain downstream of the bubble formation point is subjected to slug flow. As no models regarding the wave speed in a stratified horizontal air-water pipe flow were found in literature, the wave speed $c$ is assumed to be that of water, i.e. $c_{w}=1483 \mathrm{~m} / \mathrm{s}$ with a bulk modulus $K_{w}$ of $2.2 \mathrm{e}+09 \mathrm{~Pa}$ and density equal to $1000 \mathrm{~kg} / \mathrm{m}^{3}$ for cases $A C W C 1$ and $A C W C 5$. For case $A C W I 5, c_{w}$ is considered to be infinitely high due to the incompressible nature of the water. In contrast, the wave speed in the return bend (length $1.5 \pi D$ ) and the outlet tube (length $4 D$ ) of the tube is considerably lower than the wave speed found in both water and air, a phenomenon first observed by Mallock (1910). The model proposed by Kieffer (1977) is adopted:

$$
\begin{aligned}
c_{K}= & \frac{G_{a}}{G_{w}} \rho_{w, \text { ref }} \frac{p_{\text {ref } f} / \rho_{a, \text { ref }}}{p_{\text {out }}}+\exp \left(\frac{p_{\text {ref }}-p_{\text {out }}}{K_{w}}\right) \\
& {\left[\left\{\left(1+\frac{G_{a}}{G_{w}}\right) \rho_{w, \text { ref }}\right\}^{1 / 2}\left\{\frac{G_{a}}{G_{w}} \frac{\rho_{w, \text { ref }} p_{\text {ref } f} / \rho_{a, \text { ref }}}{p_{\text {out }}^{2}}+\frac{1}{K_{w}} \exp \left(\frac{p_{\text {ref }}-p_{\text {out }}}{K_{w}}\right)\right\}^{1 / 2}\right]^{-1}, }
\end{aligned}
$$

where $p_{\text {ref }}, \rho_{a, r e f}$ and $\rho_{w, \text { ref }}$ are chosen as 1 bar, $1.2 \mathrm{~kg} / \mathrm{m}^{3}$ and $1000 \mathrm{~kg} / \mathrm{m}^{3}$, respectively. $G$ denotes the mass flux of air (index 'a') and water (index 'w'). In the last part of the inlet tube $(1 D)$ the wave speed has some intermediate value between the liquid wave speed and the Kieffer wave speed, but it will be assumed in the model that the wave speed is equal to the Kieffer wave speed as the exact evolution of the wave speed is unknown. This means that the Kieffer wave speed is applied to a total tube length of $(5+1.5 \pi) D$. The total time 
interval $\Delta t_{\max }$ is consequently calculated as follows:

$$
\Delta t_{\text {max }, \text { model }}=4\left[\frac{4 D}{c_{w}}+\frac{(5+1.5 \pi) D}{c_{K}}\right] .
$$

The model by Kieffer (1977) is based on the work of McWilliam and Duggins (1969) and requires a number of assumptions. Firstly, it neglects the effect of surface tension on the wave speed, which is acceptable for bubbles with radii larger than 1e-06m. Secondly, Equation (10) is only valid when the flow compression and expansion happens isothermally. It was verified that the temperature in the domain stayed within $2 \mathrm{~K}$ of the initial value of $300 \mathrm{~K}$. Thirdly, a more stringent assumption is that of a uniform distribution of small air bubbles throughout the continuous water layer, such that the mixture can be considered as a homogeneous medium. Due to the size of the air bubbles in the present simulations, this condition is actually not met.

The values for $\Delta t_{\max }$, both obtained directly from the force profile and modelled as explained above, are given in Table 5 . For the cases where $p_{\text {out }}$ was set to 5 bar, the model is close to the numerical data despite the fact that not all assumptions of the underlying model are met. The value of $\Delta t_{\max }$ is similar in case $A C W C 5$ and $A C W I 5$, showing that the compressibility of the liquid only has a negligible effect.

When $p_{\text {out }}$ is equal to $1 \mathrm{bar}$, the model is not as accurate. It is noteworthy that $\Delta t_{\text {max,model }}$ is almost double of $\Delta t_{\text {max,sim }}$, especially since there is a strong effect of harmonics in the force profile (see Figure 26). Why the force profile exhibits strong harmonics when $p_{\text {out }}$ equals 1 bar, but not when $p_{\text {out }}$ is 5 bar is currently not understood.

Table 5: Period $T$ between two major force peaks, time diffence between two consecutive smaller peaks as found in the simulations $\Delta t_{\max , \text { sim }}$ and determined from a model for the wave speed $\Delta t_{\text {max }}$ model .

\begin{tabular}{|c|c|c|c|c|}
\hline Case & $T[\mathrm{~s}]$ & $\Delta t_{\text {max,sim }}[\mathrm{s}]$ & $\Delta t_{\text {max,model }}[\mathrm{s}]$ & $\frac{\left|\Delta t_{\text {max }, \text { sim }}-\Delta t_{\text {max }, \text { model }}\right|}{\Delta t_{\max , \text { sim }}}[\%]$ \\
\hline ACWC1 & 0.170 & 0.0121 & 0.02284 & 88.8 \\
ACWC5 & 0.150 & 0.0052 & 0.00496 & 4.6 \\
ACWI5 & 0.145 & 0.0055 & 0.00488 & 11.3 \\
\hline
\end{tabular}

Overall, it can be concluded that the main effect of the flow compressibility is the possibility of having pressure waves moving back and forth along the tube at a finite speed. The reflection of the pressure wave in the current domain is a numerical phenomenon related to the in- and outlet conditions, but wave reflections can occur in a real-life piping system as well. In that case, a highfrequent oscillation with a period related to the distance travelled by the wave is superposed on the force profile obtained in the incompressible flow simulations. The amplitude of this oscillation is of the same order of magnitude as the narrow force peak caused by the water layer impacting on the tube wall and only slowly decreases over time. 


\section{Flow through a deforming tube}

After having analyzed the flow profile inside a rigid U-bend, the effect of an elastic tube is investigated for three different flow rates. These cases are summarized in Table 6 . The computational time of each two-way FSI simulation is $270 \mathrm{~h}$ on 6 nodes, each containing 2 processors of type Intel E5-2680v3 (with 24 cores per node or 144 cores in total). During that time, about 0.13 s of physical flow time was calculated. One-way coupling was applied in a single case. Oneway coupling means that the pressure and shear stress are transferred from the flow solver to the structure solver, in which the corresponding displacement is calculated, but this displacement is not transferred back to the flow solver. In other words, this represents a CFD case in which, during each time step, the pressure and shear stress are used in a CSM computation.

Table 6: List of cases used in the FSI analysis. The case identifiers are listed in column 1. Column 2 identifies the CFD case defined in Table 3 on which the FSI case is based. Column 3 shows whether the case is a one-way or two-way coupled FSI simulation. The mass flux at the inlet of the domain is noted in column 4 . The inlet profile was taken from a fully-developed flow in a long tube, as discussed in Section 4.5.

\begin{tabular}{|c|c|c|c|}
\hline Case & CFD case & Type & $G\left[\mathrm{~kg} / \mathrm{m}^{2} s\right]$ \\
\hline FSI50-1W & UG50 & one-way & 50 \\
FSI50 & UG50 & two-way & 50 \\
FSI75 & UG75 & two-way & 75 \\
FSI100 & UG100 & two-way & 100 \\
\hline
\end{tabular}

\subsection{Force profile}

As mentioned in Section 4.6, varying the operating density changes the reported force profile on the tube wall in the vertical direction. When coupling the flow solver to a structural solver, a non-zero operating density would induce an error because the weight of the fluid inside the domain is misrepresented. In order to transfer a correct load to the structure solver, the operating density $\rho_{\text {ref }}$ should be set to zero. Changing the operating density does not affect the qualitative flow mechanism in the bend nor does it change the period of the force signal, but the average axial force does vary. In this section, the analysis in Section 4.6 is extended to include the axial force profile extracted from the two-way FSI simulations. The axial force profiles from the original CFD cases (i.e. with non-zero operating density), the adapted CFD cases (i.e. with zero operating density) and the two-way FSI simulations are shown in Figure 27.

The force profile of the two-way FSI cases differ in two aspects from the CFD calculations: the slight increase in time period and the high-frequent oscillation after a large force peak (moment of bubble creation). As these observations are not found in the rigid tube analysis, they must be related to the displacement of the tube. These differences arise for all mass flow rates. 


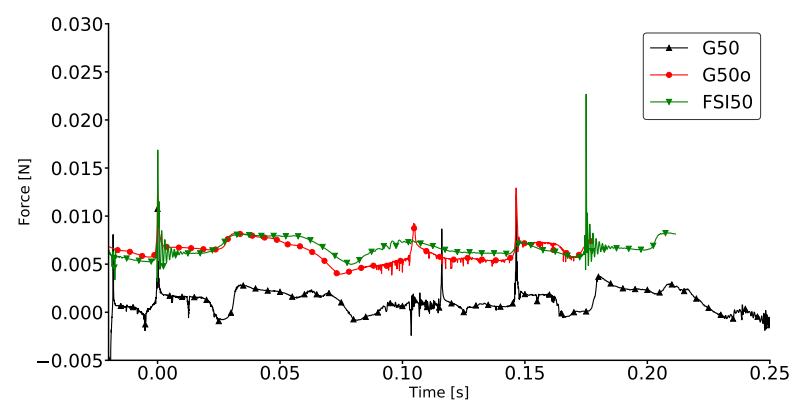

(a)

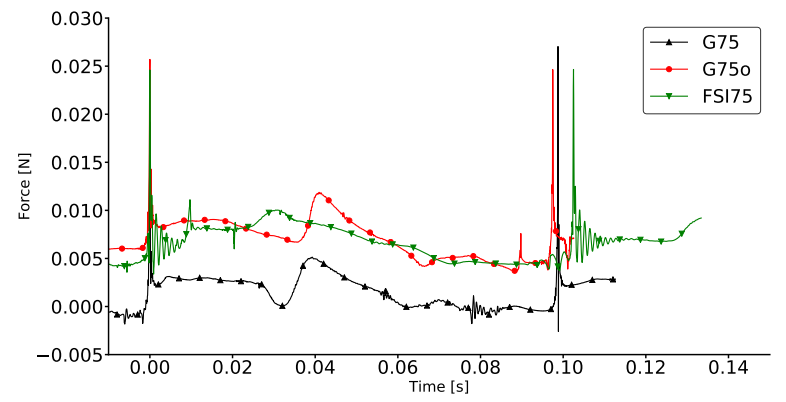

(b)

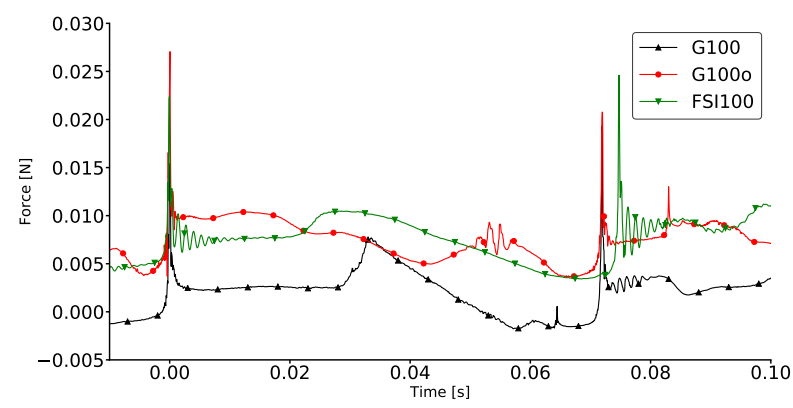

(c)

Figure 27: Axial force as a function of time for the fluid-structure interaction simulations for following mass fluxes: (a) $G=50 \mathrm{~kg} / \mathrm{m}^{2} \mathrm{~s}$, (b) $G=75 \mathrm{~kg} / \mathrm{m}^{2} \mathrm{~s}$ and (c) $G=100 \mathrm{~kg} / \mathrm{m}^{2} \mathrm{~s}$. The force profile is compared to the CFD cases with the same mass flow rate, both with and without changing the operating density. The time scales are not the same in these figures. 


\subsection{One-way or two-way coupling?}

The output of the flow solver - the force profile - is significantly changed when considering the tube displacement, as the period of the force signal as well as the oscillations after a peak were increased due to the tube's motion. The question therefore arises whether the tube motion is also different in the one-way and two-way simulations. To answer this question, the motion of a single point on the U-bend, depicted in Figure 28, is analyzed. Figure 29 shows the motion of this point, just before and after a force peak (the time scale was adapted such that a force peak corresponds to time instant zero).

Clearly, there are two major distinctions in tube motion between the one-way and two-way simulations. Firstly, the motion of the tube is severely damped in the case of the two-way simulation, yet no damping arises in the one-way FSI. No material damping was defined in the structure so all the damping should be due to energetic losses in the flow. This damping is effectively modelled in case of the strong coupling, but is absent in case of one-way coupling since the motion of the tube is not fed back to the flow solver. The energy of the vibration stays more or less constant during one period (even though there might be some decay due to numerical error). The next impact at time step 0s increases the vibration amplitude in the one-way case, but is damped in the two-way FSI simulation. Secondly, when calculating the frequency of oscillation, the frequency of vibration in case $F S I 50-1 W$ is about $1139 \mathrm{~Hz}$, which is considerably higher than $838 \mathrm{~Hz}$ which is found in case FSI50. The difference can be attributed to the absence of added mass effect in case of one-way FSI, meaning that the effective mass which is vibrating is lower. Alternatively, a linear perturbation analysis learns that the eigenfrequency of the second eigenmode equals $1154 \mathrm{~Hz}$. The mode shape corresponding to this eigenfrequency strongly resembles the vibration obtained from the one-way FSI simulation. This suggests that the second eigenmode of the structure is triggered in the one-way FSI simulation, whereas this is not the case in the two-way coupling.

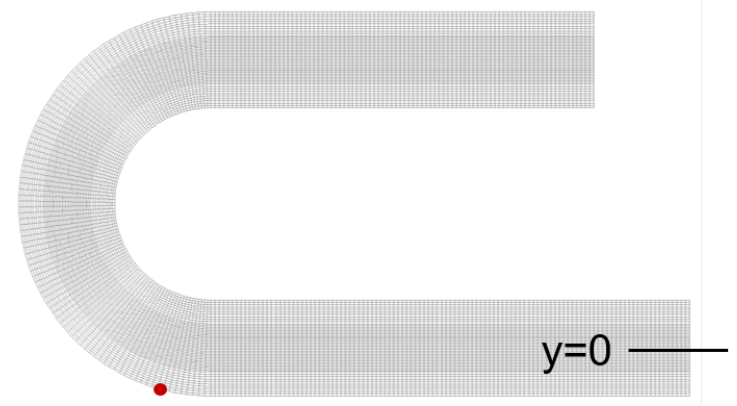

Figure 28: Initial position of the node (highlighted in red) used for determining the vibration parameters. 


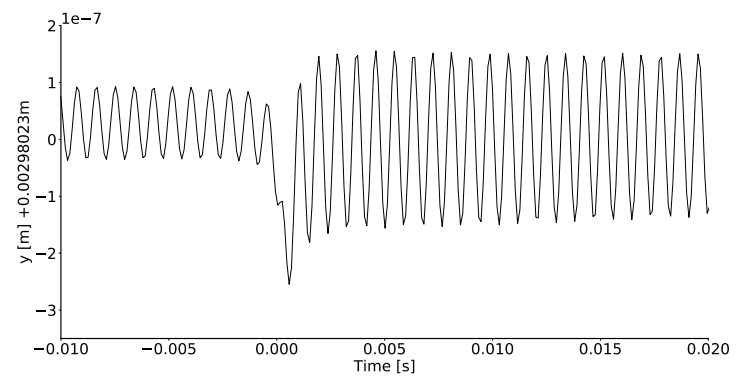

(a)

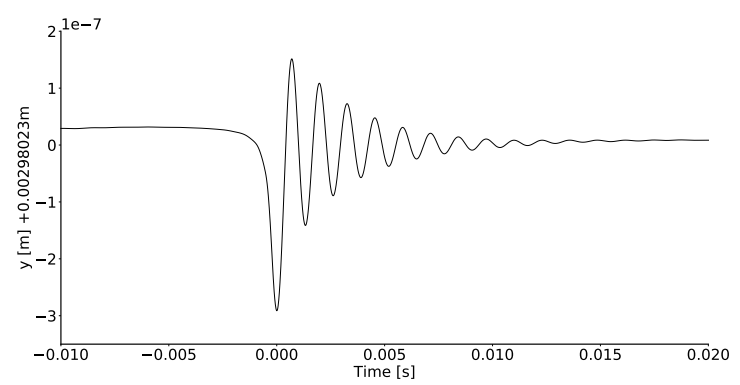

(b)

Figure 29: Motion of a single node for two cases: (a) FSI50 - $1 W$ and (b) FSI50. The horizontal axis was rescaled such that a force peak corresponds to time instant 0 s for both cases. 


\subsection{Tube displacement in two-way FSI simulations}

In this section, only the more accurate two-way FSI calculations are considered because the force equilibrium at the tube wall is enforced explicitly. As noted in Section 5.2, the tube moves only around a time instant where a force peak occurs. The subsequent high-frequent oscillation of the centerline of the U-bend following such a bubble creation is shown in Figure 30, for different instants of physical time. It should be noted that, in order to visualize the displacement of the centerline, the deformation of the centerline was scaled with a factor 10,000. It can be concluded that the bend vibrates around a mean negative displacement (due to the weight of the liquid). The force peak in the U-bend can be seen as an impact force imposed on a small area at the inner side of the bend, near the inlet. The resulting vibration is the response of the tube on this impact force. The frequency is influenced by the material stiffness, the material mass and the liquid mass inside the bend. The damping can only be due to the two-phase mixture as no material damping is modelled.

Further analysis leads to the obervation that the U-bend vibration can be described as a damped oscillation with a frequency $f_{N}$ and a damping constant $\zeta$. The values of these parameters are derived from the fitting of a damped sinusoidal oscillation on the transient displacement of a single point highlighted in Figure 28. The calculated vibration constants are presented in Table 7 for three different mass flow rates: $50 \mathrm{~kg} / \mathrm{m}^{2} \mathrm{~s}, 75 \mathrm{~kg} / \mathrm{m}^{2} \mathrm{~s}$ and $100 \mathrm{~kg} / \mathrm{m}^{2} \mathrm{~s}$. The transient displacement, as a function of the physical time calculated in the two-way FSI simulations, is shown in Figure 31 for the three analyzed mass flow rates. On each subfigure, the exponential function $\exp \left(-\zeta 2 \pi f_{N} t\right)$ is also depicted, where the amplitude and time delay have been determined from the least squares model used in the fitting of the damped sinusoidal oscillation.

Table 7: Damping constant $\zeta$ and natural frequency $f_{N}$ of the damped sinusoidal oscillation fitted to the displacement data of the node highlighted in Figure 28.

\begin{tabular}{|c|c|c|}
\hline$G\left[\mathrm{~kg} / \mathrm{m}^{2} \mathrm{~s}\right]$ & $\zeta[-]$ & $f_{N}[\mathrm{~Hz}]$ \\
\hline 50 & 0.375 & 838 \\
75 & 0.379 & 855 \\
100 & 0.386 & 856 \\
\hline
\end{tabular}

It is concluded that the natural frequency of the oscillation does not show a clear relation with the increasing mass flow rate. The values of $f_{N}$ are almost the same for the two highest mass flow rates, but a slightly lower frequency is found for $50 \mathrm{~kg} / \mathrm{m}^{2} \mathrm{~s}$. Probably, the elasticity and the density of the material has the highest influence on the natural frequency of the vibration. As these solid material properties do not change for the different cases, the exponential fitting does not yield significantly different results for different mass fluxes. However, no material damping was defined, so the damping is only due to the damping in the fluid. An influence of the mass flow rate on the damping constant is 


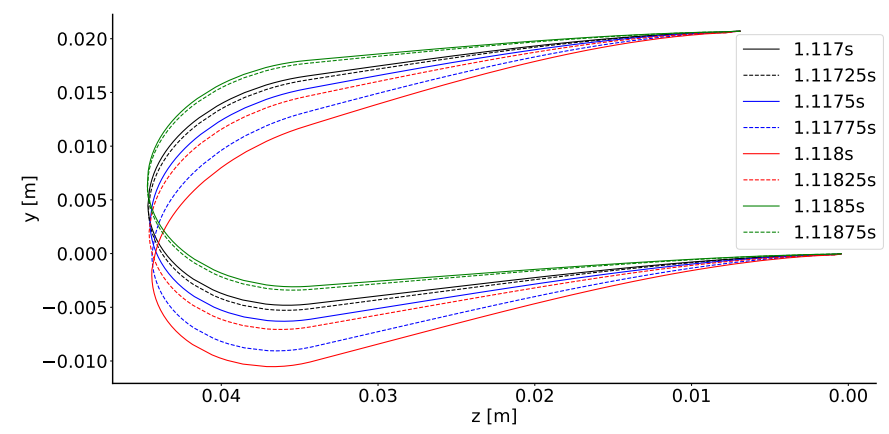

Figure 30: Location of the centerline of the U-bend, at different time instants, in case FSI 100. The deformation of the centerline is scaled with a factor equal to 10,000 in order to visualize the deformation of the entire bend in one figure.

reported in Table 7: the damping constant increases slightly but monotonously with the mass flux as expected.

\section{Conclusion}

A broad numerical investigation into the vibration caused by a two-phase flow inside a U-bend is presented in this paper. Two regimes are discerned: a gravity-dominated regime for relatively low mass flow rates and an inertiadominated regime for higher mass flow rates. Although one calculation shows the steady nature of the flow in the inertia-dominated regime, most of the research focuses on the gravity-dominated regime where the heavier water flows back inside the U-bend. This flow reversal causes an increase in water level at the inlet of the U-bend, breaking up the continuous air layer into discrete, relatively large bubbles. Moreover, this bubble creation causes a large liquid impact on the tube wall, which causes a small-amplitude, high-frequent oscillation that can be simulated and quantified.

In the first part of this research, the tube wall is rigid. The CFD simulations lead to conclusions regarding the physical nature of the flow as well as the difficulties of the numerical modelling of this type of flow. Firstly, it is shown that material parameters such as liquid viscosity and wall wetting angle have little or no effect on the flow behavior inside the U-bend. In contrast, the inlet condition, more precisely the water level imposed at the inlet, significantly affects the period of the force signal. If the incoming water fraction is set to 0.1, more high-frequent oscillations appear in the force profile due to the large space taken up by the air bubbles in the bend, possibly taking up entire crosssections at certain locations in the bend. Furthermore, the boundary condition imposed at the outlet, quantified by changing the operating density used in the flow solver, affects the average force exerted on the bend wall, but does not seem to have an effect on the period of the oscillation. The effects of inlet and outlet condition on the flow are important to note and quantify, as 


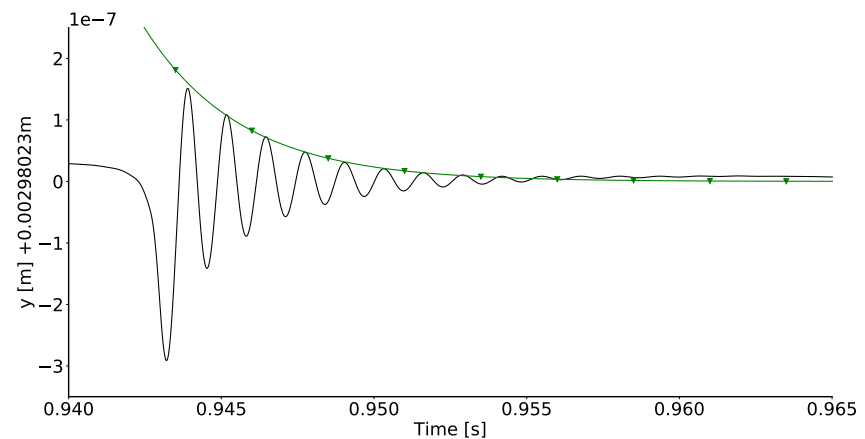

(a)

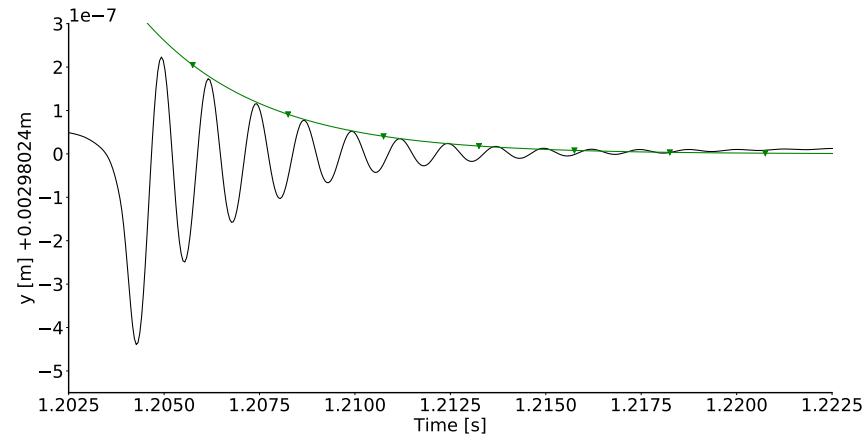

(b)

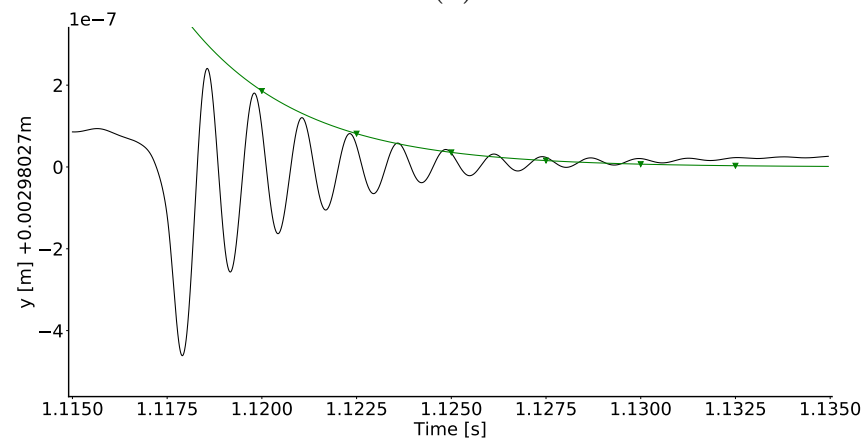

(c)

Figure 31: Displacement profile of a single node as a function of the calculated physical time for following mass fluxes: (a) $G=50 \mathrm{~kg} / \mathrm{m}^{2} \mathrm{~s}$, (b) $G=75 \mathrm{~kg} / \mathrm{m}^{2} \mathrm{~s}$ and (c) $G=100 \mathrm{~kg} / \mathrm{m}^{2} \mathrm{~s}$. The envelope represents the exponential function $\exp \left(-\zeta 2 \pi f_{N} t\right)$, with amplitude and time delay determined from the least squares model. 
the exact inlet and outlet conditions are generally not known from empirical data, where the experimental set-up is much larger than the test section and where local pressure distribution or void fraction data are not available. For future comparison between numerical and experimental analysis of vibrations, reports of transient boundary conditions are crucial in order to get a good match. Finally, the influence of the mass flow rate of a fully-developed flow on the period of the axial force is analyzed; it is found that the period of the force signal is inversely proportional to the mass flow rate, at least as long as the flow rate is sufficiently low to have flow reversal inside the bend, i.e. the gravitydominated regime. For high mass fluxes, no bubbles are formed inside the bend and consequently the pipe does not vibrate. Here, the inertia is dominant over the gravity. The distinction between the gravity- and inertia-dominated regime can be quantified with a characteristic Froude number which could only be narrowed down to a range $1.9-3.8$ with the current set of simulations. Below this value, the flow is gravity-dominated, whereas the inertia of the flow is dominant above this value.

In the second part of this research, FSI simulations are performed in order to quantify the magnitude of the vibration of the bend due to the liquid impact on the wall. The importance of setting the operating density to zero in these simulations is highlighted. Also, there is a clear distinction in the force profile and the tube vibration in a one-way and two-way FSI simulation. In the case of two-way coupling, the tube vibration resembles a damped oscillation, the parameters of which are quantified for three distinct mass flow rates. The natural frequency is not significantly affected by the incoming mass flux, which probably means the frequency is mainly determined by the solid parameters and the added mass of the fluid present in the bend. Contrarily, the damping coefficient monotonously increases with the mass flux through the U-bend. As no material damping was defined in the structural model, the damping coefficient is directly affected by the flow inside the bend.

Overall, a broad numerical investigation into the flow field inside a U-bend and the vibration caused by this internal flow has been presented in this paper. Comparison with empirical data proved difficult due to lack of precise transient profiles at the inlet and outlet of the U-bend in literature, but valuable conclusions regarding the nature of the gravity-dominated flow inside a small-diameter U-bend can be drawn from the proposed research.

\section{Acknowledgments}

The authors gratefully acknowledge the funding by the Research FoundationFlanders (FWO), through the Ph.D. fellowship of Laurent De Moerloose. The computational resources (Stevin Supercomputer Infrastructure) and services used in this work were provided by the VSC (Flemish Supercomputer Center), funded by Ghent University, FWO and the Flemish Government - department EWI. The authors also wish to acknowledge the substantial contribution to this research of the recently deceased prof. dr. ir. Jan Vierendeels to this work. 


\section{References}

Annerel, S., Claessens, T., Degroote, J., Segers, P., Vierendeels, J., 2014. Validation of a numerical fsi simulation of an aortic bmhv by in vitro piv experiments. Medical Engineering and Physics 36 (8), 1014-1023.

Belfroid, S., Nennie, E., Lewis, M., 2016. Multiphase forces on bends - large scale 6 inch experiments. SPE Annual Technical Conference and Exhibition.

Brackbill, J. U., Kothe, D. B., Zemach, C., 1992. A continuum method for modeling surface tension. Journal of Computational Physics 100 (2), 335354 .

Charreton, C., Béguin, C., Ross, A., Étienne, S., Pettigrew, M. J., 2015. Twophase damping of internal flow: Physical mechanism and effect of excitation parameters. Journal of Fluids and Structures 56, 56-74.

Da Silva Lima, R., Thome, J., 2012. Two-phase flow patterns in U-bends and their contiguous straight tubes for different orientations, tube and bend diameters. International Journal of Refrigeration 35, 1439-1454.

De Kerpel, K., Ameel, B., Huisseune, H., T'Joen, C., Canière, H., De Paepe, M., 2012. Two-phase flow behaviour and pressure drop of R134a in a smooth hairpin. Internal Journal of Heat and Mass Transfer 55, 1179-1188.

De Ridder, J., Degroote, J., Van Tichelen, K., Vierendeels, J., 2017. Predicting modal characteristics of a cluster of cylinders in axial flow: from potential flow solutions to coupled cfd-csm calculations. Journal of Fluids and Structures 74, 90-110.

De Ridder, J., Doaré, O., Degroote, J., Van Tichelen, K., Schuurmans, P., Vierendeels, J., 2015. Simulating the fluid forces and fluid-elastic instabilities of a clamped-clamped cylinder in turbulent axial flow. Journal of Fluids and Structures 55, 139-154.

Degroote, J., Bathe, K. J., Vierendeels, J., 2009. Performance of a new partitioned procedure versus a monolithic procedure in fluid-structure interaction. Computers and Structures 87, 793-801.

Degroote, J., Souto-Iglesias, A., Paepegem, W. V., Annerel, S., Bruggeman, P., Vierendeels, J., 2010. Partitioned simulation of the interaction between an elastic structure and free surface flow. Computer Methods in Applied Mechanics and Engineering 199, 2085-2098.

Geng, Y., Ren, F., Hua, C., 2012. An auxiliary measuring technology of wet gas flow based on the vibration signals of the pipe. Flow Measurement and Instrumentation 27, 113-119.

Kakaç, S., Liu, H., Pramuanjaroenkij, A., 2012. Heat Exchangers: Selection, Rating and Thermal Design. CRC Press. 
Kieffer, S., 1977. Sound speed in liquid-gas mixtures: water-air and water-steam. Journal of Geophysical Research 82 (20), 2895-2904.

Lannes, D. P., Gama, A. L., 2018. On the relationship between pipe accelerance and the void fraction of internal two-phase flow. Journal of Fluids and Structures 80, 350-369.

Lin, P. Y., Hanratty, T. J., 1986. Effect of pipe diameter on flow patterns for air-water flow in horizontal pipes. International Journal of Multiphase Flow 13 (4), 549-563.

Mack, A., Joshi, H., Belfroid, S., 2018. Numerical rebuilding of dynamic instabilities and forces in multiphase pipe bend flow. International Journal of Computational Methods and Experimental Measurements 6 (2), 358-372.

Mallock, A., 1910. The damping of sound by frothy liquids. Proceedings of the Royal Society A: Mathematical, Physical and Engineering Sciences 84, 391395.

McWilliam, D., Duggins, R. K., 1969. Speed of sound in bubbly liquids. Proceedings of the Institution of Mechanical Engineers C: Journal of Mechanical Engineering Science 184, 102-107.

Menter, F. R., 1994. Two-equation eddy-viscosity turbulence models for engineering applications. Industrial and Engineering Chemistry Research 32, $1598-1605$.

Nakamura, T., Fujita, K., Kawanishi, K., Yamaguchi, N., Tsuge, A., 1995. Study on the vibrational characteristics of a tube array caused by two-phase flow. part 1: random vibration. Journal of Fluids and Structures 9, 519-538.

Pettigrew, M. J., Janzen, V. P., 2016. Two-phase flow-induced vibration: some unanswered questions? 11th International Conference on Flow-Induced Vibration.

Pettigrew, M. J., Taylor, C. E., 2003a. Vibration analysis of shell-and-tube heat exchangers: an overview - part 1: flow, damping, fluidelastic instability. Journal of Fluids and Structures 18, 469-483.

Pettigrew, M. J., Taylor, C. E., 2003b. Vibration analysis of shell-and-tube heat exchangers: an overview - part 2: vibration response, fretting-wear, guidelines. Journal of Fluids and Structures 18, 485-500.

Pettigrew, M. J., Taylor, C. E., Fisher, N. J., 1998. Flow-induced vibration: recent findings and open questions. Nuclear Engineering and Design 185, 249276 .

Tzotzi, C., Bontozoglou, V., Andritsos, N., Vlachogiannis, M., 2011. Effect of fluid properties on flow patterns in two-phase gas-liquid flow in horizontal and downward pipes. Industrial and Engineering Chemistry Research 50, 645-655. 
Ubbink, O., 1997. Numerical prediction of two fluid systems with sharp interfaces. Ph.D. thesis, University of London.

Usui, K., Aoki, S., Inoue, A., 2012. Flow behavior and pressure drop of twophase flow through c-shaped bend in vertical plane. Journal of Nuclear Science and Technology 55, 1179-1188.

Wang, C.-C., Chen, I. Y., Huang, P.-S., 2005. Two-phase slug flow across small diameter tubes with the presence of a vertical return bend. Internal Journal of Heat and Mass Transfer 48, 2342-2346.

Wang, C.-C., Chen, I. Y., Lin, Y.-T., Chang, Y.-J., 2008. A visual observation of the air-water two-phase flow in small diameter tubes subject to the influence of vertical return bends. Chemical Engineering Research and Design 86, 12231235 .

Weaver, D. S., Fitzpatrick, J. A., 1988. A review of cross-flow induced vibrations in heat exchanger tube arrays. Journal of Fluids and Structures 2, 73-93.

Yetisir, M., Weaver, D. S., 1986. The dynamics of heat exchanger U-bend tubes with flat bar supports. Journal of Pressure Vessel Technology 108, 406-412. 